\title{
Design and Testing of a Prototype Beam Absorber for the PXIE MEBT
}

\author{
Curtis Baffes ${ }^{\mathrm{a}}$, Alexander Shemyakin ${ }^{\mathrm{a}}$ \\ ${ }^{a}$ Fermi National Accelerator Laboratory, \\ PO Box 500, Batavia, IL 60510, USA \\ E-mail: cbaffes@fnal.gov, shemyakin@fnal.gov
}

\begin{abstract}
One of the goals of the PXIE at Fermilab is to demonstrate the capability to form an arbitrary bunch pattern from an initially CW $162.5 \mathrm{MHz} \mathrm{H}$ - bunch train coming out of an RFQ. The bunch-by-bunch selection will take place in the $2.1 \mathrm{MeV}$ Medium Energy Beam Transport (MEBT) by directing the undesired bunches onto an absorber that needs to withstand a beam power of up to $21 \mathrm{~kW}$, focused onto a spot with a $\sim 2 \mathrm{~mm}$ rms radius. A design was developed to respond to the driving requirements: high surface power density of $17 \mathrm{~W} / \mathrm{mm}^{2}$ and resistance to ion-induced surface blistering. A $1 / 4$ - size prototype of the absorber was manufactured, and its thermal properties were tested with an electron beam generating a peak power density similar to the one expected during normal operation of the PXIE beam line. The note describes the absorber concept, the prototype, the testing procedure with the electron beam, and the results of testing.
\end{abstract}




\section{Contents}

1. Introduction $\quad 2$

2. Comparison with similar devices $\quad 4$

3. PXIE design concept 6

$\begin{array}{ll}3.1 \text { Constraints } & 6\end{array}$

$\begin{array}{lr}3.2 \text { Design considerations } & 6\end{array}$

3.2.1 Vacuum 6

$\begin{array}{ll}\text { 3.2.2 Surface effects - blistering and sputtering } & 7\end{array}$

3.2.3 Modular Design Concept 9

3.2.4 Cooling 9

3.2.5 Conceptual PXIE Design Implementation 12

4. Prototype Design 14

$\begin{array}{ll}4.1 \text { Motivation } & 14\end{array}$

$\begin{array}{ll}4.2 \text { Configuration } & 14\end{array}$

$\begin{array}{ll}4.3 \text { Manufacturing } & 16\end{array}$

$\begin{array}{ll}\text { 4.4 Preliminary Analysis } & 17\end{array}$

5. Test results $\quad 20$

$\begin{array}{ll}5.1 \text { The test stand } & 21\end{array}$

$\begin{array}{ll}5.2 \text { Energy deposition } & 21\end{array}$

5.3 Beam imaging and the beam footprint profile 24

5.4 Optical Temperature Measurements 25

$\begin{array}{ll}5.5 \text { Thermocouple Temperature Measurements } & 27\end{array}$

5.6 Analysis of Test and Correlation Results $\quad 29$

$\begin{array}{ll}5.7 \text { Cooling Studies } & 31\end{array}$

6. Conclusions $\quad \mathbf{3 4}$

6.1 Lessons Learned $\quad 34$

6.2 Summary and Top-Level Conclusions 36

\section{Introduction}

Fermilab considers upgrade scenarios for the injection complex, and the leading version is an SRF linac [1] that is nearly identical to the first stage of the previously developed proposal called Project X [2]. The concept for the front end of this future machine will be tested by carrying out an R\&D program known, for historical reasons, as the Project $\mathrm{X}$ Injector Experiment (PXIE) [3]. PXIE consists of an H- ion source; a Low Energy Beam Transport (LEBT); a CW 2.1-MeV RFQ; a Medium Energy Beam Transport (MEBT); two SRF 
cryomodules operating at 2K; a High Energy Beam Transport (HEBT); and a beam dump (Figure 1).

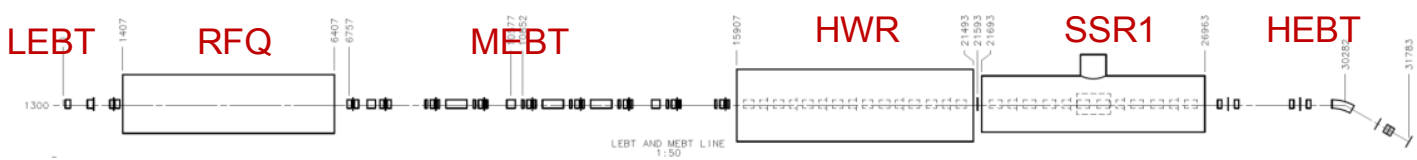

Figure 1: PXIE layout

One of the key functions of the MEBT [4], [5] is to form a required arbitrary bunch structure from the CW beam provided by the RFQ. This is accomplished by a chopping system, where wide-band kickers direct the undesired bunches to the MEBT absorber (Figure 2).

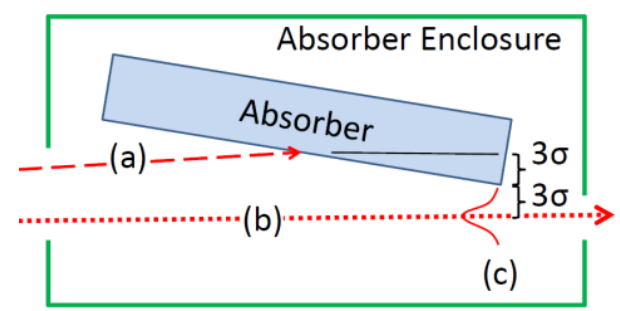

Figure 2: Schematic representation of the MEBT absorber, showing (a) chopped beam, (b) passed beam, (c) beam profile and $6 \sigma$ transverse shift between the centers of the chopped and passed beams.

In the nominal scenario of operation, the chopping system creates a beam pattern with time average current (over $\sim 1 \mu \mathrm{s}$ ) of $1 \mathrm{~mA}$ from the initially $\mathrm{CW} 5 \mathrm{~mA}$ beam, hence directing $4 \mathrm{~mA}$ to the absorber. Considering possible test and upgrade scenarios, the absorber is designed for the maximum RFQ design current of 10mA. Detailed beam parameters for the absorber are shown in Table 1.

Table 1: MEBT absorber requirements

\begin{tabular}{|l|l|}
\hline Species & $\mathrm{H}-$ \\
\hline Energy & $2.1 \mathrm{MeV}$ \\
\hline Beam structure from RFQ & $165.2 \mathrm{MHz} \mathrm{CW}$ \\
\hline Nominal current to absorber & $4 \mathrm{~mA}$ \\
\hline Maximum current to absorber & $10 \mathrm{~mA}$ \\
\hline Maximum incident power & $21 \mathrm{~kW}$ \\
\hline Nominal RMS beam transverse size & $\sigma_{\mathrm{x}}=\sigma_{\mathrm{y}}=2 \mathrm{~mm}$ \\
\hline Length of the absorber vacuum enclosure & $650 \mathrm{~mm}$ flange-to-flange \\
\hline Vacuum (beam on) & $\leq 1 \mathrm{E}-6$ Torr \\
\hline
\end{tabular}

Challenges presented by the absorber design include maintaining vacuum quality, managing surface effects (sputtering and blistering), containing secondary particles, accommodating radiation effects, and the survival of temperatures and temperature-induced mechanical stresses.

In this paper, we describe the present concept of the PXIE MEBT absorber and results of testing a prototype with an electron beam. Section 2 compares requirements for this absorber with similar existing devices. Section 3 describes the design's constraints and concept. A prototype that was design and manufactured to test this concept is the subject of Section 4 . The 
prototype was installed at a test stand and irradiated by an electron beam with representative power density. We present results of these tests in Section 5 and conclude in Section 6.

\section{Comparison with similar devices}

The challenges associated with intercepting a high power density proton/ion beams apply to several types of devices commonly used in accelerators: targets, beam dumps, collimators, and chopper absorbers such as the subject of this paper. We will consider several examples to underscore specifics of the PXIE case.

First, let us note that one key factor differentiating beam intercepting devices is the particle energy. For energies of order $10 \mathrm{MeV}$ and above, penetration of the beam into the absorbing material could be millimeters or more. In this case, energy deposition occurs volumetrically, i.e. in three dimensions. In contrast, for energies below a few $\mathrm{MeV}$, penetration is shallow, and energy deposition may behave as a surface phenomenon.

Second, it is important whether an absorbing device is absorbing energy in a pulsed mode or continuously. If the in-pulse temperature rise is comparable with the average temperature increase, the effect of temperature cycling may be dominant, and modeling the depth of the penetration and 3-dimensional thermal fields during energy deposition is required. However, if the energy deposition is continuous (e.g. provided by a CW source), the details of 3-dimensional heating and transient energy storage become less important. In such a case, the assumption that heating is a 2-dimensional surface phenomenon may be appropriate.

For the beam conditions of the PXIE MEBT absorber, $\mathrm{H}$ - ions at $2.1 \mathrm{MeV}$ incident on a molybdenum surface penetrate a few tens of microns along the beam direction. Given the grazing angle of incidence, median implantation depth from the surface is $<1 \mu \mathrm{m}$. This is on the same order as the surface roughness average for a typical machined surface. Any attempt to model 3-dimensional heating would need to acknowledge the specific surface roughness characteristics of the absorber. For the purposes of this note, energy deposition on the PXIE MEBT absorber is regarded as 2-dimensional surface heating.

A number of devices subject to surface energy deposition have been described in literature. The most obvious analogs are absorbers (called also targets, dumps, or stops) of other MEBT chopping systems, reviewed in [6]. However, in comparing devices one relevant metric is surface power density.

CERN's Linac4/SPL chopper dump ([7], [8]) is designed to accept up to $2.5 \mathrm{~kW}$ of $3 \mathrm{MeV}$ $\mathrm{H}$ - ions. The absorbing surface is a cone surrounding the beam line, and the beam is incident on the axisymmetric surface at a grazing angle of incidence. The design condition is for the pulsed beam with up to $2.8 \mathrm{~ms}$ duration repeating at $50 \mathrm{~Hz}$. Design surface power density is reported as $3 \mathrm{~W} / \mathrm{mm}^{2}$ (averaged over $1 \mathrm{~s}$ ). Within the macro-pulse, power density would be $21 \mathrm{~W} / \mathrm{mm}^{2}$ (average over 2.8ms macro-pulse duration). The core of this device is shown in Figure 3. The design utilizes hypervapotron cooling channels. Note that in other applications, hypervapotron cooling has been used to manage surface power densities at least as high as $15 \mathrm{~W} / \mathrm{mm}^{2}$ [9].

The Spallation Neutron Source MEBT chopper target intercepts $2.5 \mathrm{MeV}$ proton beam, with $1 \mathrm{~ms}$ long macro-pulses at $60 \mathrm{~Hz}$ ([10], [11]). This target includes a curved surface inclined to the beam. Surface power density (average over $1 \mathrm{~s}$ ) is approximately $3 \mathrm{~W} / \mathrm{mm}^{2}$. Surface power density (average over $1 \mathrm{~ms}$ macro-pulse) is in excess of $50 \mathrm{~W} / \mathrm{mm}^{2}$. Power over a $1 \mathrm{~ms}$ long pulse is $8.4 \mathrm{~kW}$. This type of pulse-mode absorber presents different challenges than a $\mathrm{CW}$ device. For example, the design must possess sufficient thermal capacitance in the beam interaction region to limit heating during the macro-pulse, and often 3-dimensional heating must 
be modeled to assess transient effects [11]. Additionally one must design for survival of billions or more transient thermal cycles and their associated cyclic stresses.

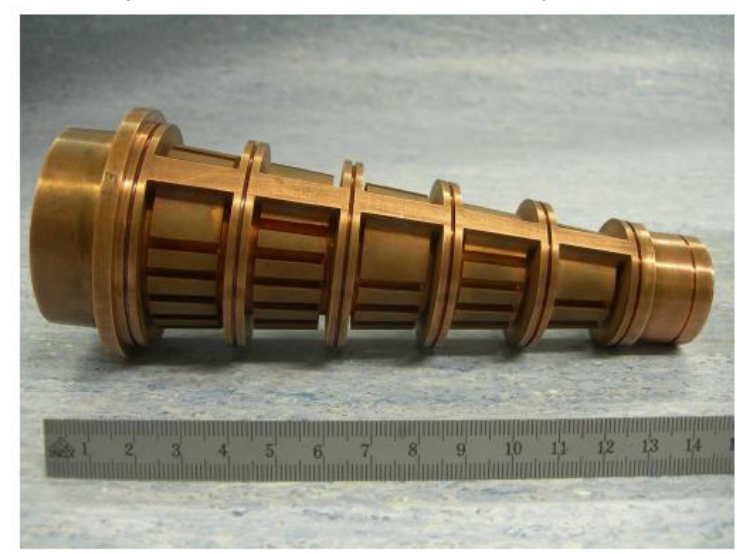

Figure 3: CERN Linac4/SPL Chopper Dump Core [7]

Another type of an intercepting device is a beam dump installed at the end of the beam line. In contrast to the chopper absorbers, in this case the beam size can be significantly increased to lower the surface power density. For instance, Los Alamos National Lab built an ogive-shaped beam stop for use in its Low Energy Demonstration Accelerator (LEDA) [12]. This beam stop was designed to intercept a $670 \mathrm{~kW} \mathrm{CW}$ beam of $6.7 \mathrm{MeV}$ protons. The beam strikes the $>2 \mathrm{~m}$ long axisymmetric absorbing surface at a grazing angle of incidence. The combination of beam and surface geometry results in manageable peak surface power density of $2.1 \mathrm{~W} / \mathrm{mm}^{2}$.

In comparison with these examples, the main challenge for the PXIE MEBT absorber is a significantly higher average power density (Table 2). In part, it is defined by the choice of the transverse focusing structure that was optimized, first of all, for the kicker performance and the beam emittance growth [13]. The choice essentially fixed the longitudinal space available to the absorber and the beam size. Note that beam sweeping in horizontal direction (with the individual bunches being kicked vertically) across the absorber surface had been considered as a means to decrease the average deposited power density but was discarded because of added complexity and contradiction with the present scheme where the absorber is immediately followed by a differential pumping system.

Table 2: Comparison of incident surface power density

\begin{tabular}{|l|c|c|c|}
\hline Device & $\begin{array}{c}\text { Beam } \\
\text { Structure }\end{array}$ & $\begin{array}{c}\text { Surface Power Density } \\
\text { (average over } \mathbf{1 s} \text { ) }\end{array}$ & $\begin{array}{c}\text { Macro-Pulse } \\
\text { Surface Power Density } \\
\text { (average over } \mathbf{1 m s} \text { ) } \\
\text { W/mm }\end{array}$ \\
\hline $\begin{array}{l}\text { LEDA beam stop } \\
{[12]}\end{array}$ & CW & 2 & 2 \\
\hline $\begin{array}{l}\text { LINAC4/SPL } \\
\text { Chopper Dump [7][8] }\end{array}$ & Pulsed & 3 & 50 \\
\hline $\begin{array}{l}\text { SNS Chopper Target } \\
{[10][11]}\end{array}$ & Pulsed & 3 & 21 \\
\hline PXIE MEBT Absorber & CW & 21 & 21 \\
\hline
\end{tabular}


With using all of the available longitudinal space to decrease the grazing angle, the design peak incident power density at the surface of the PXIE MEBT absorber is $21 \mathrm{~W} / \mathrm{mm}^{2}$ (average on all time scales $\geq 1 \mu \mathrm{s}$ ).

In conclusion, the primary and differentiating characteristics the PXIE MEBT Absorber application are:

- CW operation

- Energy deposition very near the surface, well approximated as 2-dimensional surface heating

- Comparatively high surface power density (average over all time scales $\geq 1 \mu \mathrm{s}$ ), driven by longitudinal space constraints.

These characteristics and constraints led us to the PXIE design concept that will be described in the next section.

\section{PXIE design concept}

In describing the design concept for the PXIE MEBT absorber, we will first introduce broad constraints (Section 3.1), and then explore detailed design considerations. Specific considerations include the need to maintain vacuum along the beamline (Section 3.2.1), the need to minimize damage to the absorbing surface (Section 3.2.2), a modular-design concept to limit fabrication complexity and risk (Section 3.2.3), the design of water cooling for the removal of absorbed power (Section 3.2.4), and finally a conceptual description of how these design considerations would be combined in a final PXIE design (Section 3.2.5).

\subsection{Constraints}

As described above, the chosen system architecture requires a relatively short absorber that must accept beam with small transverse size. The system design also imposes constraints on vacuum within the absorber. We assume that $\mathrm{H}$ - ions in the absorbed beam will recombine to form $\mathrm{H}_{2}$ gas. This gas load must be efficiently pumped to maintain vacuum better than $10^{-6}$ Torr within the absorber enclosure. Because the beam is $\mathrm{CW}$, any vacuum events (for example "bursts" of gas trapped in the absorber surface) are likely to disturb the propagating beam. SRF cryomodules are located just downstream of the absorber, so any absorber vacuum events that would affect the cryomodules must be avoided.

\subsection{Design considerations}

\subsubsection{Vacuum}

Vacuum along the beam path better than $10^{-6}$ Torr is required to minimize losses in the propagating beam. As mentioned above, the primary gas load is expected to be $\mathrm{H}_{2}$ formed from beam ions. For a $10 \mathrm{~mA}$ absorbed beam, the gas load is $Q_{H_{2}}=9.6 \cdot 10^{-4}$ Torr $\cdot 1 / \mathrm{s}$. In order to handle this large gas load, the absorber enclosure is pumped with four turbo pumps with a total effective pumping speed of $w_{a b s}=3000 \mathrm{l} / \mathrm{s}$. The resulting pressure within the absorber $P_{a b s}$ can be estimated as:

$$
P_{a b s}=\frac{Q_{H_{2}}}{w_{a b s}}=\frac{9.6 \cdot 10^{-4} \mathrm{Torr} / \mathrm{s}}{3000 \mathrm{l} / \mathrm{s}}=3.2 \cdot 10^{-7} \mathrm{Torr}
$$


In order to achieve the design pumping speed, good conductance must be maintained between the absorber surface and the pumps. For this reason, "closed" designs, such as a cone surrounding the pass-through beamline, were not pursued.

\subsubsection{Surface effects - blistering and sputtering}

Associated with surface energy deposition is the risk of surface damage by sputtering or blistering. Sputtering - the ejection of atoms from the surface - has been estimated to occur at a rate of $<1 \mu \mathrm{m} /$ year for this device [14]. As such, sputtering is not considered a design driver.

Blistering, in contrast, is. The blistering phenomenon is well documented in literature, e.g. [15], [16]. When ions are implanted in a shallow layer just below the surface, they may combine to form gas. If the ions or gas molecules are not able to diffuse away from the surface at a rate equal to the implantation rate, the gas molecules may coalesce into bubbles. These bubbles then grow, eventually rupturing through the surface and causing surface damage. Individual blisters have diameters on the order of a few $\mu \mathrm{m}$. However, heavy blistering may result in macroscopic spalling of the surface. We see three possible negative effects:

- The absorber relies on a grazing angle of incidence to limit power density. Surface roughing creates the risk of local areas developing more normal angles of incidence. This, in turn, could lead to higher energy deposition in local areas and melting or failure.

- The SRF cryomodules are susceptible to damage by particulate contamination, and great care is taken to build and operate these units using low-particulate practices. As blisters rupture, there is a risk that particulate debris will be created and then migrate into an SRF cavity. One mechanism for this transport is via gas during venting or evacuation of the vacuum chamber. This mechanism will be mitigated by employing the conventional technique of mass-flow-controlled evacuation and vent-up. In addition, the dust particles can be captured by the space charge of the $\mathrm{CW}$ beam similar to what had been observed in storage rings [17] and then drift toward SRF. If experiments show that this channel is important, we may consider an electrostatic barrier in front of the SRF section.

- As individual blisters rupture, a pressure wave would propagate into vacuum. Because of small transverse spacing between the absorber surface and the nominal beam axis, these vacuum bursts could degrade the primary beam. In the simplest model (Figure 4), the beam loss is determined by the product of pressure $P_{a b s}$ and length along the beam $L_{a b s}$. The tolerable value of the product in the steady state determines the required absorber pumping speed so that

$$
P_{a b s} \cdot L_{a b s}=3.2 \cdot 10^{-7} \text { Torr } \cdot 0.65 \mathrm{~m}=0.21 \mathrm{Torr} \cdot \mathrm{m}
$$

If a discrete blister with high internal pressure were to rupture adjacent to the propagating beam, the beam would pass through a local region of higher pressure. An order-ofmagnitude estimate of this affect can be made as follows. Assume a spherical gas blister with a $2 \mu \mathrm{m}$ radius (a typical size of experimentally observed blisters) rupturing at an internal pressure of $200 \mathrm{MPa}$ (a notional ultimate strength). The gas initially within the blister would be liberated, and would propagate hemispherically. Assume that the blister is at the downstream end of the absorber (i.e. as close as possible to the center of the propagating beam, $6 \mathrm{~mm}$ ). As the pressure burst propagates, the length of the path that the beam takes through the burst grows, but the average pressure decreases in inverse proportion with the volume of the burst. Based on the given simplified assumptions, the pressure*length product (evaluated at the center of the propagating beam) peaks at $\sim 0.5$ 
Torr $\mathrm{m}$, when the pressure burst radius has reached a radius of $\sim 7 \mathrm{~mm}$. A mitigating factor is the fact that pressure bursts would propagate quickly, and by the time it reaches a radius of $\sim 30 \mathrm{~mm}$ the pressure*length product would have dropped by an order of magnitude. Still, taking into account the very approximate nature of the estimation, this effect can contribute significantly to instantaneous beam loss and, consequently, to operation stability.

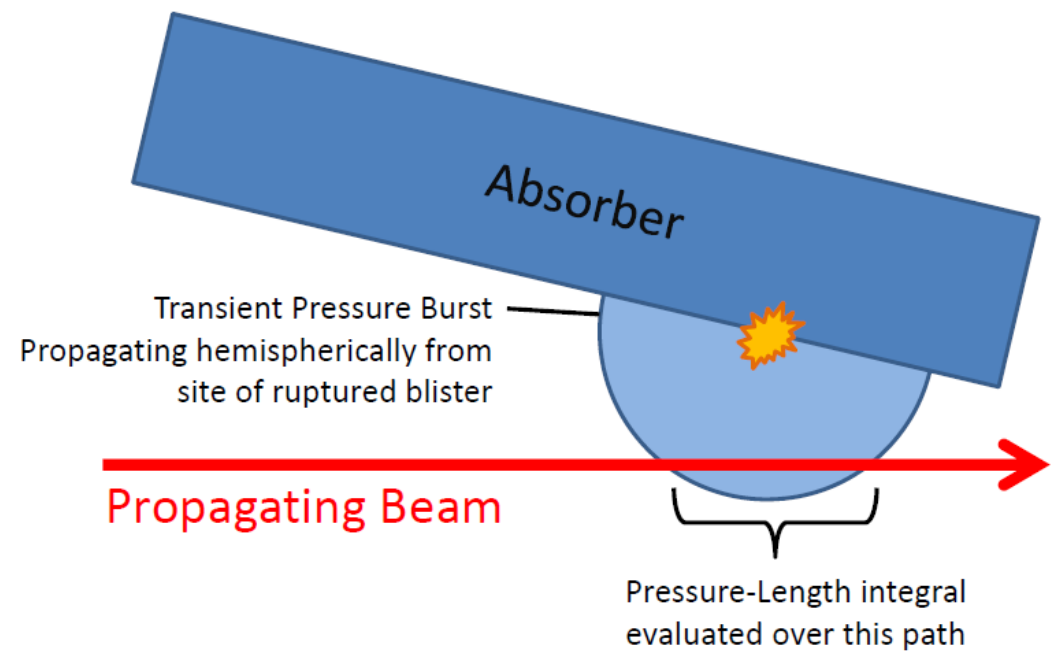

Figure 4: Blister rupture effect on beamline vacuum

Given these risks, avoiding blistering is a strong design driver. Though there is a large amount of empirical blistering data in literature, the wide variety of test conditions makes direct comparisons difficult. To summarize general trends, blistering is more severe when:

- Total particle fluence exceeds a material-specific characteristic threshold (or order $10^{20}$ particles $/ \mathrm{m}^{2}$ or greater, depending on material and irradiation parameters)

- Current density is high (of order $10 \mathrm{~A} / \mathrm{m}^{2}$ ).

- Particle energy is low (or angle of incidence is grazing). In either case, high ion concentrations are possible because implantation depth relative to the surface is small.

- Hydrogen solubility and diffusion rates in the target metal are low.

- Metal temperature is low, because higher temperatures increase diffusion rates.

- The incident surface is smooth, due to the fact that there is less free surface area for ion desorption.

In this application, the current density, particle energy and angle of incidence are constrained. In order to avoid blistering, a blistering resistant material was chosen: molybdenum alloy TZM. There are materials that offer better blistering resistance than TZM due to higher hydrogen solubility (e.g. Vanadium, Tantalum [15]). However, TZM offers more blistering resistance than conventional materials (e.g. $\mathrm{Cu}$ ) [18], [19] combined with reasonable mechanical properties and reasonable availability and cost.

The blistering threshold of TZM is reported by [18] and [19] to be $>10^{24}$ particles $/ \mathrm{m}^{2}$. Given the geometry of the absorber and a $10 \mathrm{~mA}$ beam, at the center of the beam footprint this threshold would be reached in $\sim 5$ hours of beam time. If the system is able to achieve steadystate diffusion and desorption in that time, one would not expect blistering to occur. 
In addition to blistering resistance, TZM possesses other mechanical properties favorable to this application. Most significantly, it offers high-temperature capability, with a recrystallization temperature of $\sim 1400^{\circ} \mathrm{C}$.

Blistering could be a significant effect, with consequences to the performance and the life of the absorber. A representative ion beam is not economically available for testing of this effect. As such, the current approach is to use blistering-resistant Molybdenum alloy TZM, and defer final retirement of this risk to PXIE.

\subsubsection{Modular Design Concept}

Given the choice of TZM as the material, and the complex machining of the chosen solution, the following bear consideration:

- TZM is not inexpensive, $>\$ 200 / \mathrm{kg}$ depending on form, size and grade

- TZM is brittle and prone to damage during manufacture

- Complex fabrication (to be described in subsequent sections) carries substantial risk of scrap and failure

Given these risks, a modular solution is beneficial. The $0.5 \mathrm{~m}$ length of the absorber is divided into four identical modules. These modules could be fabricated, assembled and tested individually, and then assembled together into a full-up absorber. In the event of fabrication failure, an individual module could be scrapped without extreme consequences. This philosophy was followed in developing a conceptual design for PXIE.

\subsubsection{Cooling}

Given the decision to employ a modular design, coolant flow at the module level was considered. Preliminary thermal analysis indicated that aggressive cooling was necessary to maintain acceptable surface temperatures. The chosen cooling strategy was as follows:

- Monolithic design - cooling water flows through the TZM material to avoid thermal contact resistances

- Transverse flow - coolant moves transverse to the beam direction to limit alongstream heating

- Sub-mm-scale cooling channels - cooling channels are narrow to enhance heat transfer, with a fine pitch to increase surface area available for cooling.

Through an iterative design process, the cooling scheme shown in the Figure 5 was developed. The primary driver of the cooling design was the avoidance of the transition boiling regime (and associated degradation of heat transfer). The criteria used (as suggested in [20]) were:

- Surface temperatures at the cooling channel should be $<\sim 130^{\circ} \mathrm{C}$

- Heat flux to coolant should be $\leq 1 \mathrm{~W} / \mathrm{mm}^{2}$ 


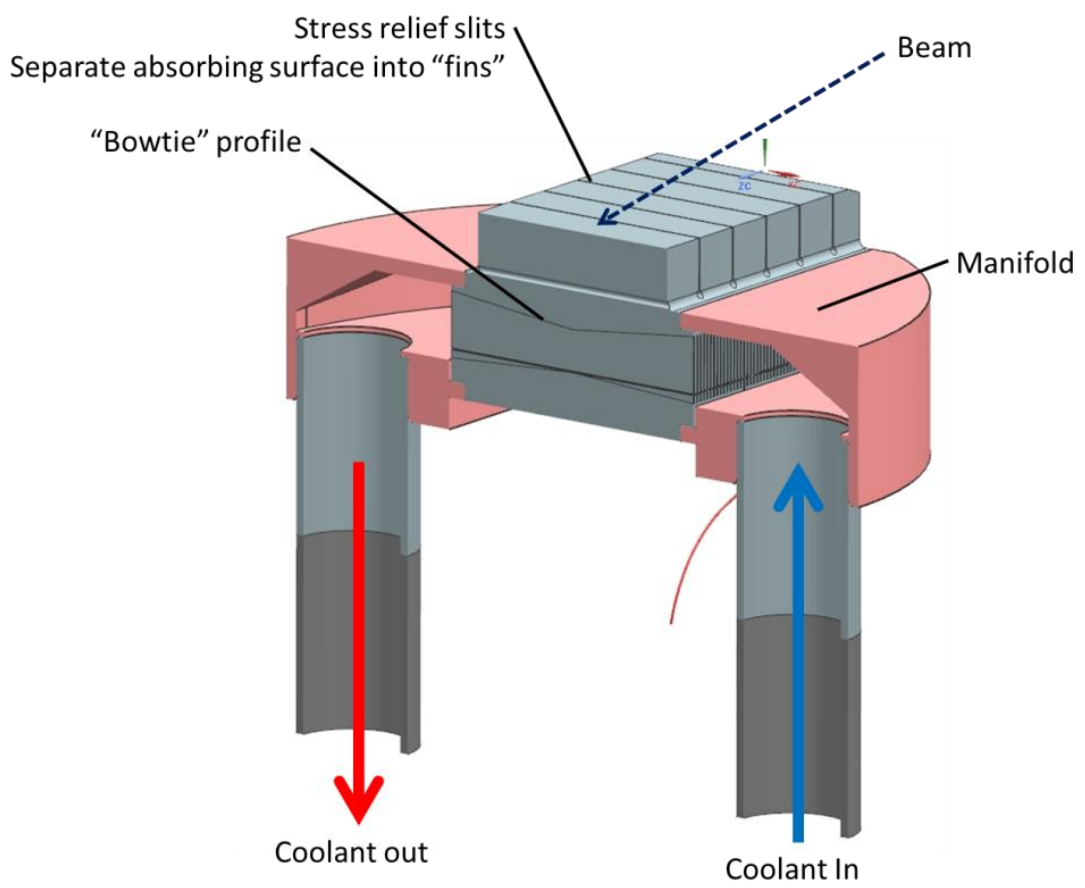

Figure 5: Single-Module cooling scheme shown in cross section: narrow channels with transverse coolant flow

For laminar flow in a narrow channel, the heat transfer coefficient $h$ can be estimated as follows [20]:

$$
h=N u \cdot k_{c} / D_{e f f}
$$

where:

$\mathrm{Nu}$ is the Nusselt number

$k_{c}$ is the thermal conductivity of the coolant

$D_{\text {eff }}$ is the effective or hydraulic diameter of the cooling channel, calculated as

$$
D_{\text {eff }}=4 * \text { Area/Perimeter }
$$

Since heat transfer is inversely proportional to $D_{\text {eff, }}$ by minimizing $D_{\text {eff }}$ one can increase heat transfer. This is accomplished by having the cooling channels very narrow in one dimension. Conventional Wire Electro-Discharge Machining (EDM) using a relatively robust 0.010 " wire produces a cut width of approximately $300 \mu \mathrm{m}$. Designing around this manufacturing process, cooling channels with $300 \mu \mathrm{m}$ width, $\sim 10 \mathrm{~mm}$ height and $1 \mathrm{~mm}$ spacing were implemented. Cooling channels fabricated in the prototype absorber are shown in the figure below. In addition to the advantage of small cut width, the use of 5-Axis CNC EDM offers the ability to implement a profile shape along the length of the channel. A "Bowtie" shape was implemented, as can be seen in the cross-section of Figure 5. This shape allows for more uniform heat transfer along the length of a given channel.

In PXIE, the footprint of the beam on the absorber surface is long $(\sim 0.4 \mathrm{~m})$, and depending on its position a given module could have strong heating at one end, in the middle, or both. The absorbing surface is separated by stress-relief slits (see Section 3.2.5), visible in Figure 5 and Figure 6. A side-effect of this feature is that longitudinal thermal conduction is substantially interrupted, i.e. almost all heat flow occurs transverse to the beam direction. As such, it is 
necessary for the cooling channels to offer similar performance along the length of the module. Fluid flow through one module was assessed with ANSYS. A set of example results is shown in Figure 7. For 5gpm flow through a single module, flow velocities vary from channel-tochannel by a factor $\leq \sim 2$. As flow rate increases, so does flow uniformity.

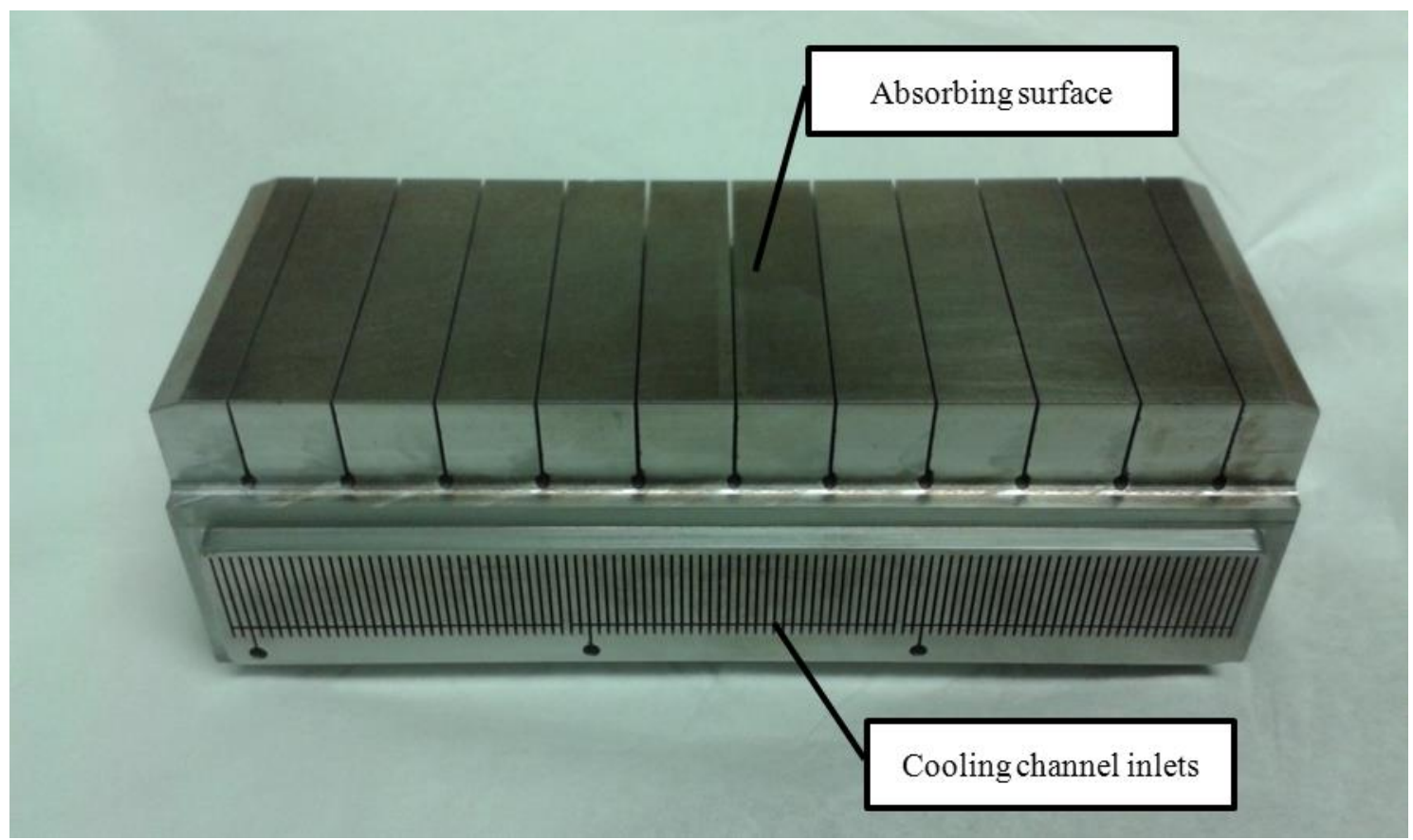

Figure 6: Prototype absorber body, prior to brazing. $300 \mu \mathrm{m}$ wide $X 1 \mathrm{~mm}$ pitch cooling channels are visible.

Within the expected range of single-module flow rates (2-10 gpm), flow through the channels is expected to be laminar (Reynold's number at 10gpm $R e=2073$, whereas turbulent transition would begin at $R e=\sim 2300$ [20]). In the laminar regime, the Nusselt number (and therefore the convection coefficient $\mathrm{h}$ ) does not depend on flow velocity. For a high-aspectration cooling channel, empirical correlations from [20] recommend $N u=6.0$, the corresponding value of the convection coefficient is $h=6.5 \cdot 10^{3} \mathrm{~W} / \mathrm{m}^{2} / \mathrm{K}$. 


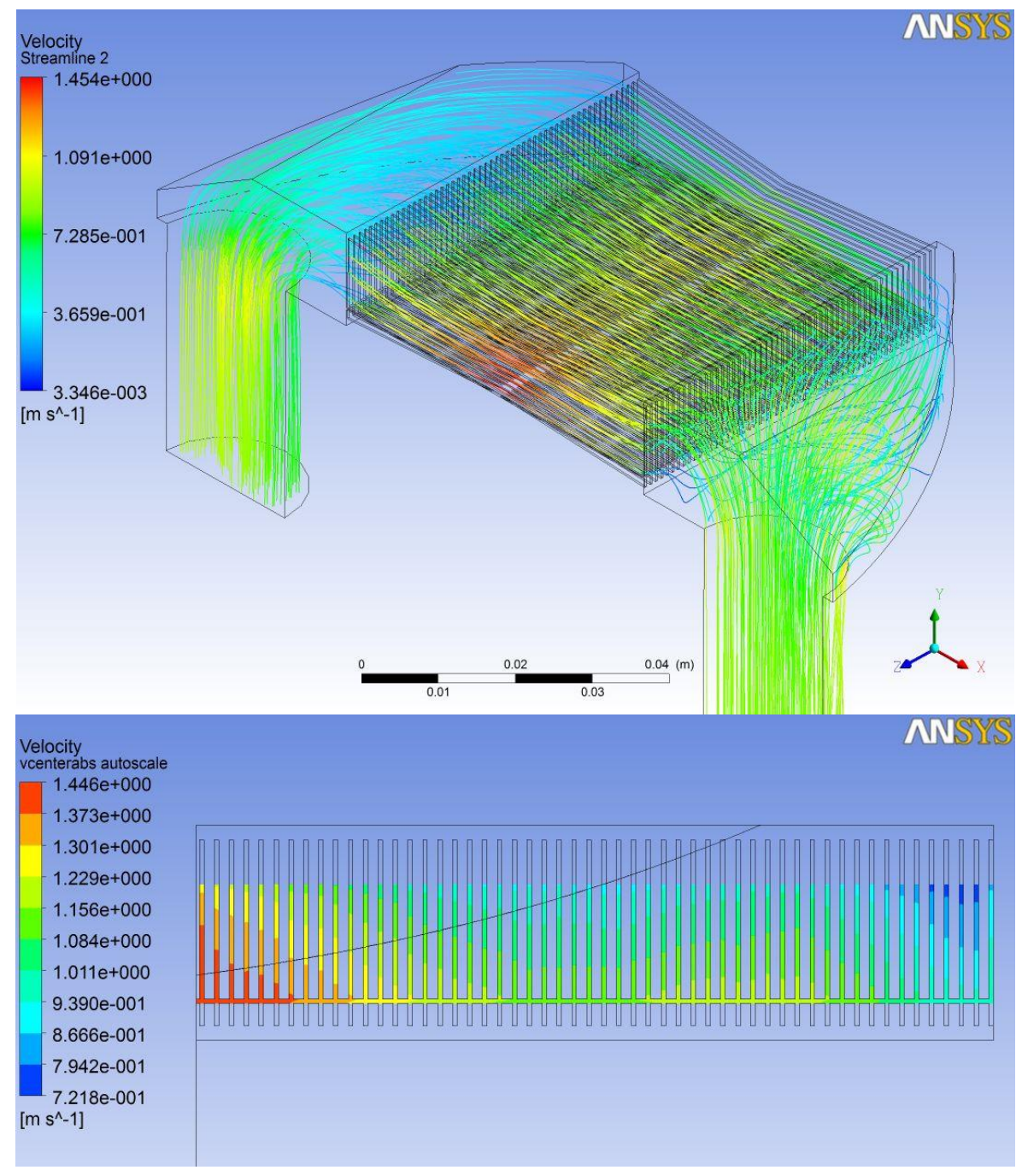

Figure 7: Fluid flow through an absorber module, $5 \mathrm{gpm}$ flow condition. Top: velocity streamline through the half-symmetric model. Bottom: velocity contour at a cross-section through the midplane.

\subsubsection{Conceptual PXIE Design Implementation}

A conceptual design for the PXIE MEBT absorber is shown in Figure 8. The four absorber modules are mounted on a common structure. This structure is adjustable in tip, tilt and piston relative to a handling flange. The modules and handling flange are then installed in a vacuum enclosure. The vacuum enclosure also accommodates four turbo pumps, which together provide 30001/s pumping speed.

Below the vacuum enclosure is an optical system which can image the absorbing surface. Visible-spectrum thermal radiation is used to approximate temperatures and indicate beam location and structure.

The modular design approach results in discontinuities in the absorbing surface. The intersection between modules must be designed to prevent beam from striking any surface at near-normal angle of incidence. This condition would result in much higher power densities and could lead to melting or failure. Additional discontinuities are present in the absorber 
surface in the form of transverse stress-relief slits. This stress relief scheme was proposed by Lebedev and Hassan et al. [21]. These slits allow for expansion of the hot absorber surface in the longitudinal direction, and reduce stresses and bowing-deflections that would otherwise occur.
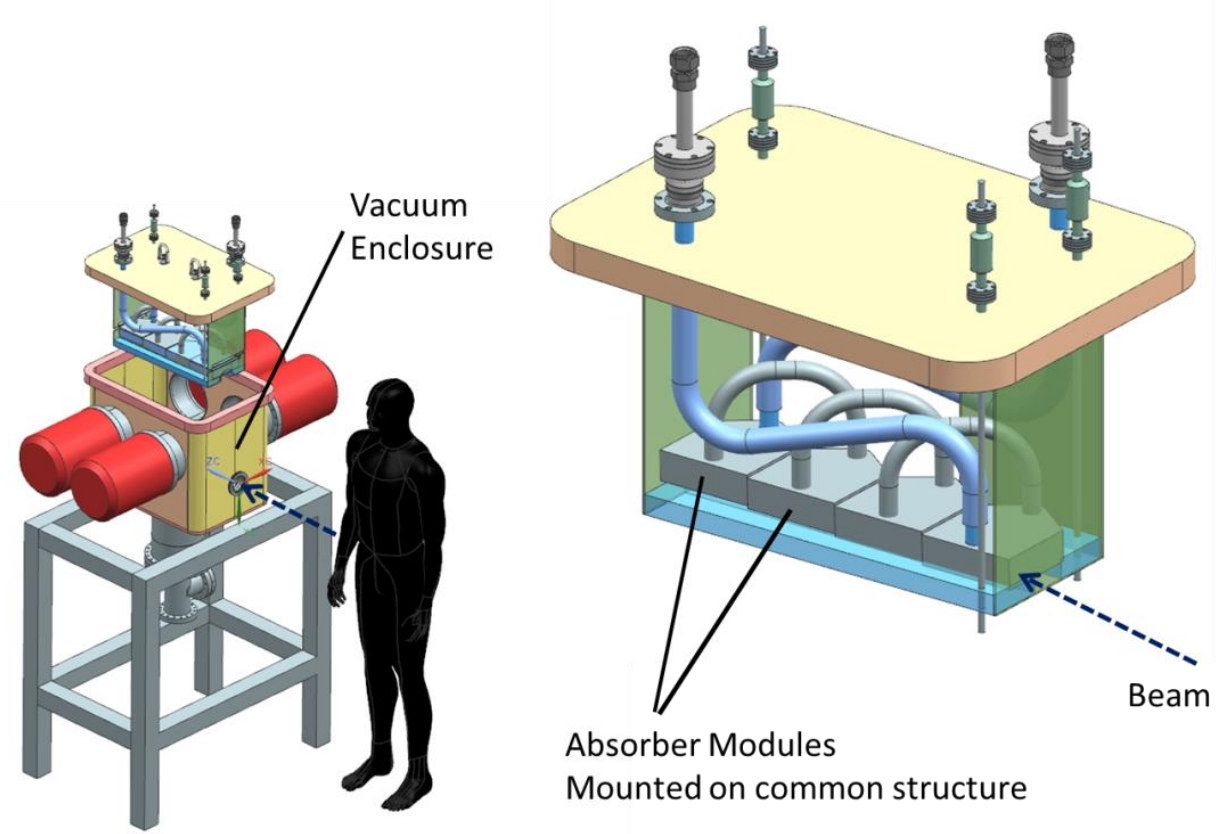

Figure 8: 4-module configuration (right), exploded view of absorber subassembly and vacuum enclosure (left)

In order to ensure that beam incidence is always grazing, a stair-step shadowing scheme [21] is implemented, as shown in Figure 9. Any features that could intercept the beam at nearnormal angle of incidence lie in the shadow of an upstream portion of the absorber.

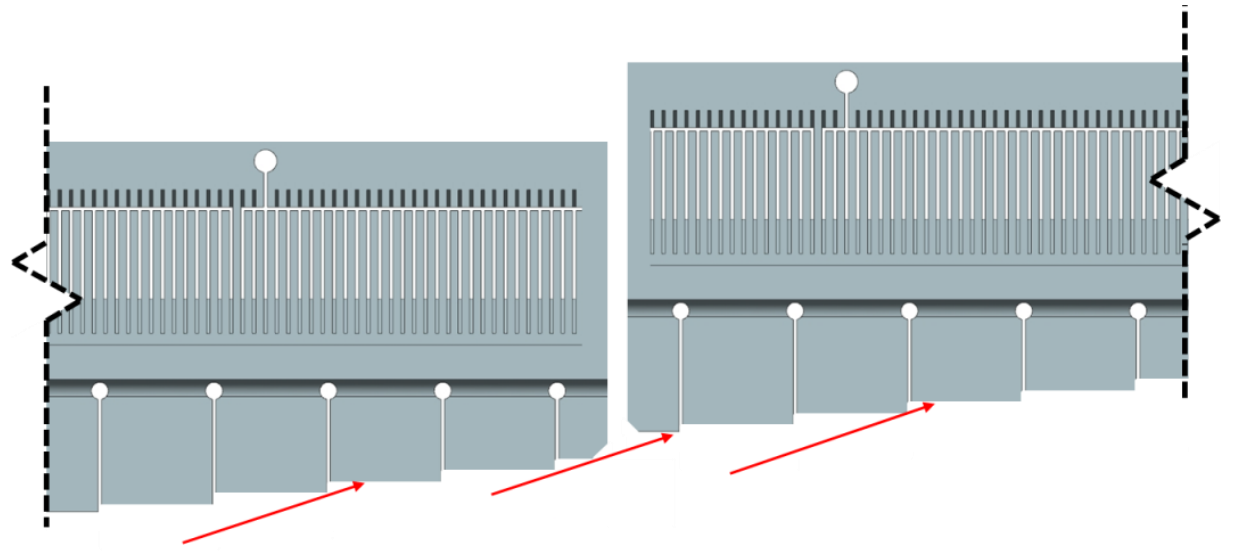

Module 1

Module 2

Figure 9: Stair-step scheme within and between modules. The vertical location of the absorbing surface is incremented such that discontinuities on the absorbing surface are shadowed. Vertical scale greatly exaggerated. 
In conclusion, a concept was developed to respond to the requirements, constraints and challenges of this application. The key features of this concept for the PXIE MEBT absorber are:

- An inclined surface which the beam strikes at grazing angle of incidence

- The absorbing surface is open (rather than a surface of revolution) to allow for good conductance of the beam-induced gas load away from the absorbing surface

- Vacuum is maintained in the $10^{-7}$ Torr range by multiple turbo pumps achieving a total pumping speed of $3000 \mathrm{l} / \mathrm{s}$.

- The absorbing surface is made from the molybdenum alloy TZM, which combines resistance to beam-induced blistering (a primary consideration), favorable hightemperature mechanical properties, and a reasonable cost.

- Aggressive cooling is provided by $300 \mu \mathrm{m}$ wide, $10 \mathrm{~mm}$ high, $1 \mathrm{~mm}$ pitch water channels machined by EDM directly in the TZM material

- To limit fabrication complexity and risk, the $\sim 0.5 \mathrm{~m}$ length of the absorbing surface is split into four modules, each $\sim 0.12 \mathrm{~m}$ in length.

This concept was deemed to entail enough complexity and risk to merit the construction of a prototype. This prototype is described in the next section.

\section{Prototype Design}

In describing the prototype design, we will first touch on the motivations for building a prototype (Section 4.1). We will then describe the prototype configuration, both in its commonalities and differences with the PXIE design (Section 4.2). The manufacturing processes used to produce the prototype are described in Section 4.3. In Section 4.4, the thermal finite element model used for prototype design and testing correlation is described.

\subsection{Motivation}

In developing the PXIE design, the following risks were identified:

- Complicated fabrication of absorber modules

- High power density and resulting aggressive thermal conditions

- Accuracy of analysis of flow and temperature conditions

- Blistering of the absorber surface in $\mathrm{H}$ - beam

The risk of blistering could only be retired in a representative ion or proton beam, which was not economically available. However, an electron test bench capable of producing representative power density (further described in Section 5.1) was available for retrofit. This motivated a prototype cycle, with the goals of retiring risks associated with fabrication, thermal conditions, and analysis.

\subsection{Configuration}

Given the modular design approach, it was practical to build and test one full-scale module. The module mounted to an 8 " OD Conflat flange, and packaged such that it could be inserted into the 8 " vacuum cross available in the test stand.

In order to replicate the peak power density of PXIE within the constraints of the test bench, the angle of incidence between the prototype absorber surface and the electron beam is more normal ( $\sim 0.16 \mathrm{rad}$ prototype vs. $\sim 0.03 \mathrm{rad}$ PXIE). Depending on beam focusing, as few as four fins (i.e. the areas on the surface partitioned by the stress relief slits) are illuminated by 
beam. However, because the stress relief slits interrupt longitudinal thermal conduction, this is adequate to characterize thermal performance of the device.

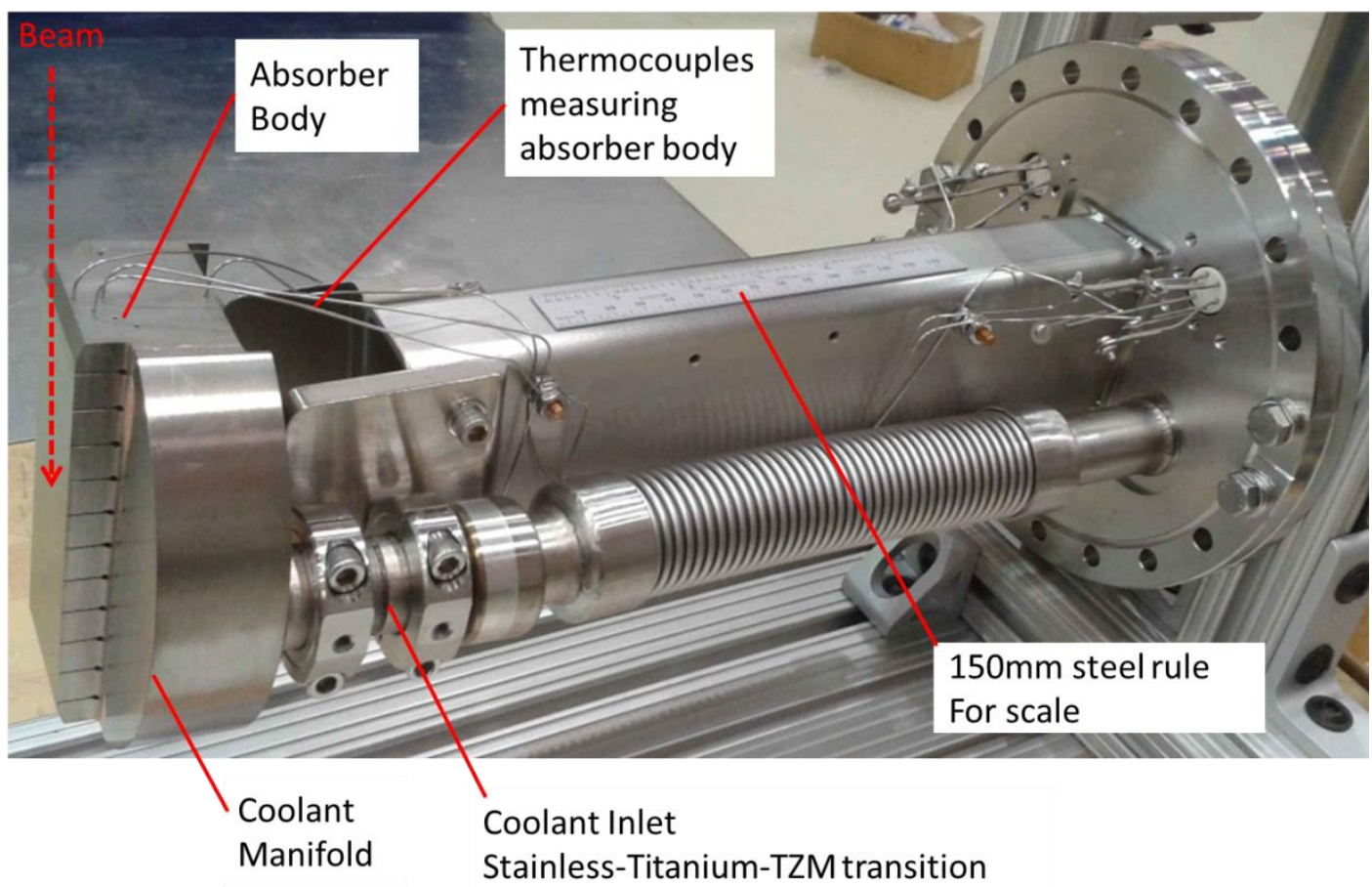

Figure 10: Prototype Absorber

The prototype is instrumented with 6 thermocouples. (Omega P/N SCAXL-032U-18-RP$\mathrm{CC}$, mineral-insulated, Type $\mathrm{K})$. These thermocouples were inserted in long holes EDM-drilled into the TZM material below the absorber surface. Thermocouples were installed in different locations within the absorber volume in order to provide temperature distribution information for use in analysis correlation. Four of the 6 thermocouples were installed below the $5^{\text {th }}$ fin, as shown in Figure 11.

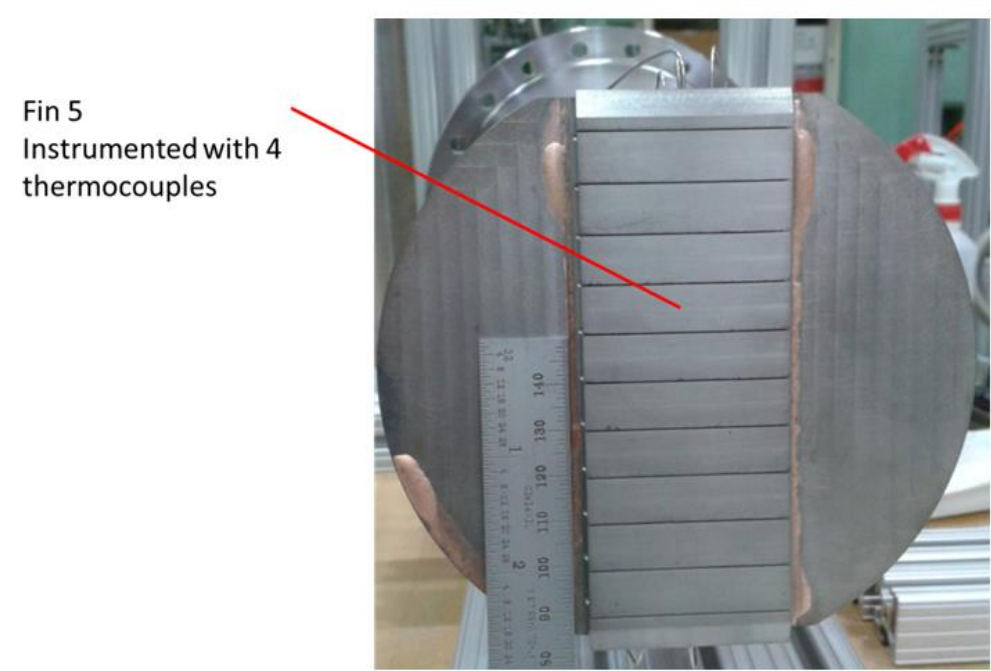

Figure 11: Location on surface beneath which the majority of thermocouples are located (See also Table 4). This area of the surface was subjected to the peak of the beam profile during testing. 


\subsection{Manufacturing}

The TZM material presents manufacturing challenges. It is brittle, and must be machined and handled with great care. It is challenging to join by welding or brazing. Two grades of TZM are available - alloy 363 is vacuum arc melted, alloy 364 is a powder-metallurgy product. Alloy 364 has inferior mechanical properties (strength and ductility), but lower cost. For the prototype cycle, the risk of using alloy 364 was accepted.

The machining of TZM components was accomplished using a combination of EDM (which is unlikely to damage the brittle material due to the absence of cutting forces) and conventional machining using carbide tooling. Conventional machining resulted in a small but acceptable level of edge chipping. Later in the manufacturing process, a small crack $(3 \mathrm{~mm}$ long, <10 $\mu \mathrm{m}$ width) was discovered through the bulk of one of the TZM manifolds during an in-process leak check. It is not known when this crack developed, but it is speculated that it was either created or exposed during a conventional machining process. The crack was drilled out and plugged during brazing. After machining, all TZM components were electro-polished to remove subsurface damage and blunt sharp features that could become failure initiation sites.

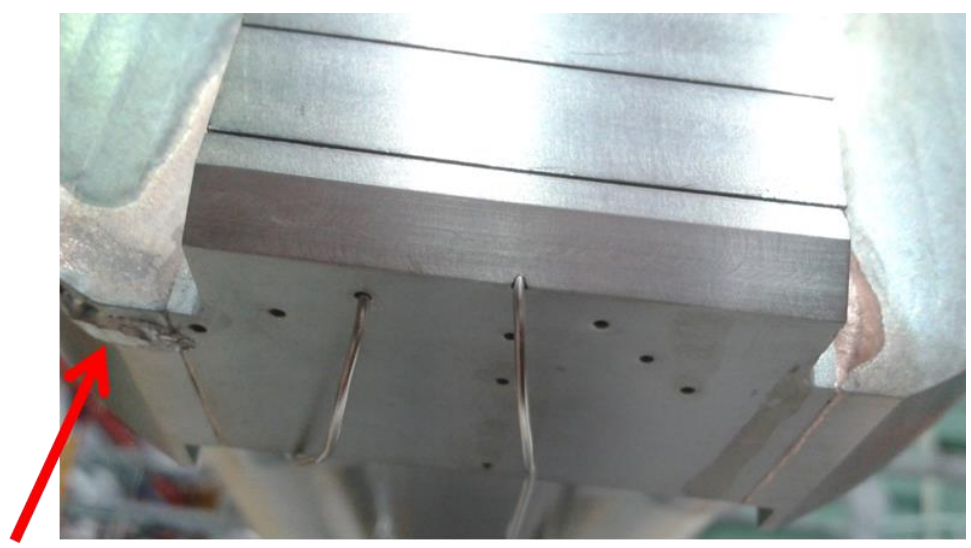

Figure 12: Site of drilled and plugged crack on final assembly

In order to have coolant in direct contact with the TZM, material transitions from TZM to a plumbing-friendly material were required. In the PXIE implementation, it is envisioned that much of the in-vacuum interconnect plumbing would be titanium. For convenience in the test bench, it was decided to transition to stainless steel. This was accomplished with a multi-step in-vacuum transition approach as shown in the Figure 13 below.

Process development was required for the TZM-to-TZM braze joints and for the TZMto-titanium E-beam weld.

Molybdenum (and low-concentration alloys such as TZM) is mutually soluble with many of the elements in groups 4-6 of the periodic table (e.g. Ti, Nb, W). Of these, Titanium is the most readily available. A procedure was developed at vendor Sciaky Welding to join the TZM tube to the titanium tube by e-beam welding. After a few iterations, complete penetration was achieved with minimal heating of surrounding material. The resulting welds were examined optically, and then subjected to leak and pressure testing. The completed TZM/Ti tubes were then ready for brazing into the TZM assembly. 


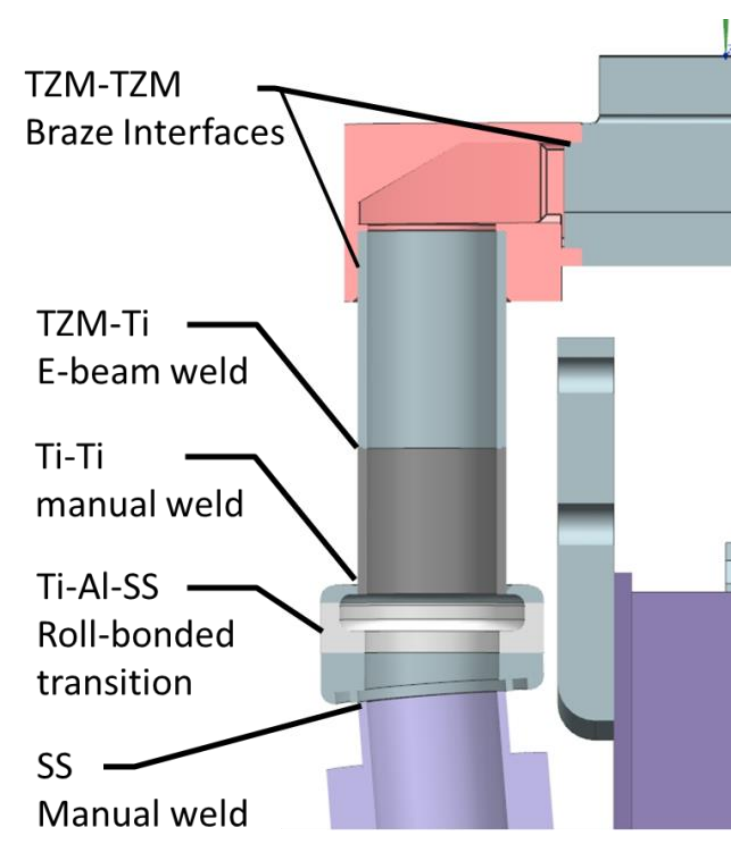

Figure 13: TZM-to-stainless transition approach

TZM is known to be difficult to wet with braze alloy, though it has been successfully brazed using Palcusil 25 [10]. Because there are two TZM-to-TZM braze joints, it is desirable to use two different brazing alloys to allow a simpler, decoupled setup of each joint. The hightemperature braze is accomplished first, and the low-temperature braze second. Braze tests were conducted with a variety of alloys and brazing conditions. Palcusil 25 in vacuum offered superior wetting, ad was used for the tube-to-manifold joint where capillary flow was required. Au35-Cu65 paste in $\mathrm{H}_{2}$ atmosphere was used for the body-to-manifold joint. The Au35-Cu65 did not wet the surface well, and was applied as a paste across the interface surface before brazing. The large amounts of alloy required for this resulted in cosmetic issues, but a sound joint was achieved. The multiple braze cycles caused some grain grown in the Titanium; grains were visible with a $\sim 5 \mathrm{~mm}$ characteristic size. This would tend to decrease the strength of the titanium, but in this application the effect proved to be of little consequence.

The brazed assembly was manually TIG welded, and the completed system successfully passed leak and pressure testing. In summary, the fabrication was successful, and aided by thorough in-process quality assurance. The presence of a crack through the bulk of the TZM was disconcerting, and gives one pause in considering the fabrication a quantity of four of these assemblies.

\subsection{Preliminary Analysis}

In order to design the PXIE and prototype absorbers, several finite element models were built in ANSYS [22]. Heat transfer is not significant in the manifolds or water lines; only the central "body" portion of the absorber, containing fins and fine water channels, was represented in any of the models.

In the PXIE case, the beam is assumed to be purely Gaussian, and the footprint of the beam on the absorber is long $(\sim 0.4 \mathrm{~m})$. As such, the rate of change of energy deposition along the length of the absorber is relatively small: fin-to-fin variation are $<80 \mathrm{~W}$, and $<10 \mathrm{~W}$ near the peak of the profile (see Figure 14). 


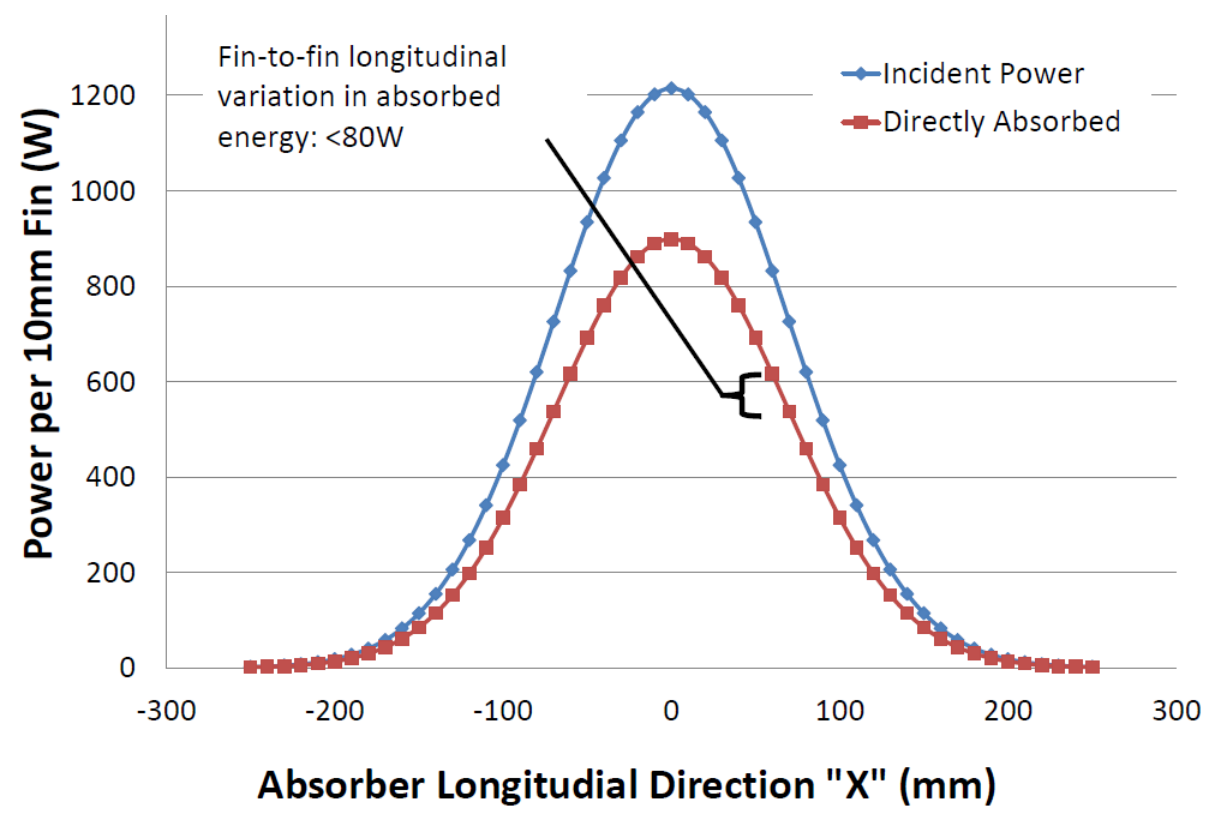

Figure 14: Longitudinal variation in energy deposition: fin-to-fin differences are $<80 \mathrm{~W}$, and $<10 \mathrm{~W}$ at the hottest fins.

As such, longitudinal heat transfer is only a minor effect. This is especially true considering the thermal interruptions created by the fin structure. Neglecting any longitudinal conduction, a simple half-symmetric "single fin" model was constructed. This model was subjected to a heat load calculated assuming a Gaussian transverse beam profile in the PXIE case, and uniform transverse beam profile in the test bench case. This model is adequate for the PXIE case, and was useful for some prototype analyses. A sample result from this model is shown in Figure 15.

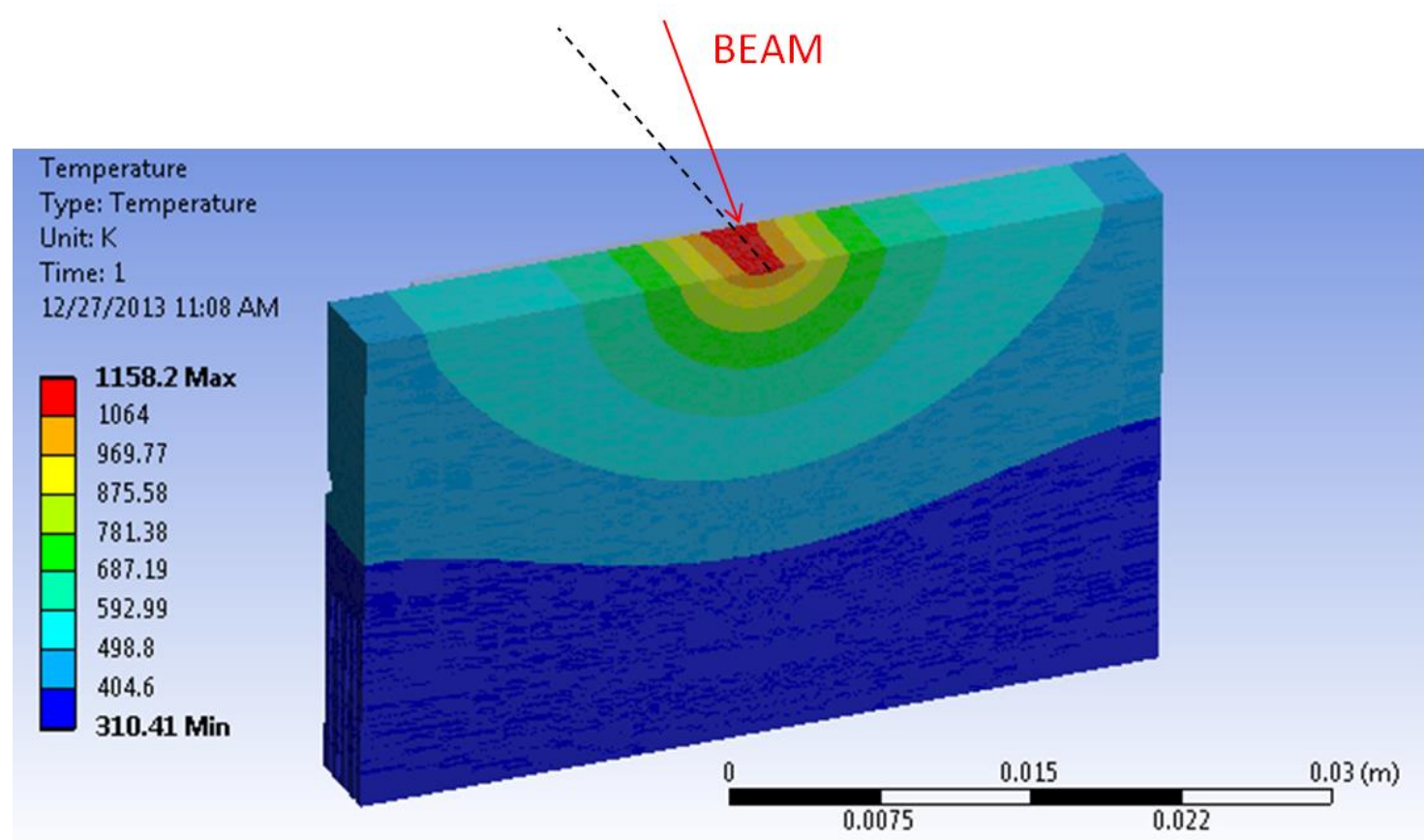

Figure 15: Single-fin finite element model. PXIE case - Gaussian beam, $\sigma x=\sigma y=2 \mathrm{~mm}, 29 \mathrm{mrad}$ angle of incidence, $17 \mathrm{~W} / \mathrm{mm} 2$ surface heat flux at peak of Gaussian profile 
However, in the course of attempting to correlate with prototype data, several model deficiencies were noted and corrected. Only the final incarnation of the model will be described here. Two of these deficiencies are worth noting here as lessons learned:

- For the single-fin model, the underlying assumptions broke down in some cases. With OTR studies to be described in Sections 5.3 and 5.4, it was determined that the beam profile contained significant structure, and that the assumption of beam uniformity, between fins and even within a single fin, was not valid. As such, it was necessary to model the full transverse width of the absorber (i.e. to not assume transverse symmetry), and at least as many fins as were illuminated by the beam.

- A further deficiency of the single-fin model is that, though heat flow between fins in the absorber is minimal, heat flow along the sheath of the thermocouple itself was enough to affect thermocouple readings. This too will be described in more detail in the test results section (see Figure 25). However, a multi-fin model was necessary to properly recover the spatial-average temperature reported by the thermocouples.

The final version of the FEA model is shown in Figure 16 and Figure 17. Features of the model are as follows:

- Model represents a longitudinal distance of four fins, large enough accommodate the peak-power-density test bench beam.

- Manifolds and the portion of the absorber body below the coolant channels are not represented. This was validated by demonstrating that heat transfer falls to nearzero values at the boundaries of the cooling channels.

- Simple convection representation: convection is modeled as occurring with a constant film coefficient of $h=6.5 \mathrm{E} 3 \mathrm{~W} / \mathrm{m}^{2} / \mathrm{K}$ and a constant far-field fluid temperature of $19^{\circ} \mathrm{C}$.

- Fully temperature-dependent thermal and mechanical properties of Mo TZM, as given in [23], are incorporated in the material model.

- The mesh is finely refined on the absorbing surface to provide $\sim 100 \mu \mathrm{m}$ transverse element size near the peak of the energy deposition. This small mesh size allows mapping of the heat load with resolution driven by the $0.24 \mathrm{~mm}$ pixel size of the camera used to determine the test bench beam profile.

- Energy deposition is a surface heat flux applied to the absorbing surface, with measured beam structure (as determined in the testing) mapped onto the model using the ANSYS workbench mapping utility.

- Analyses demonstrated that radiation from the absorbing surface to have a negligible effect, radiation was neglected in the majority of the runs.

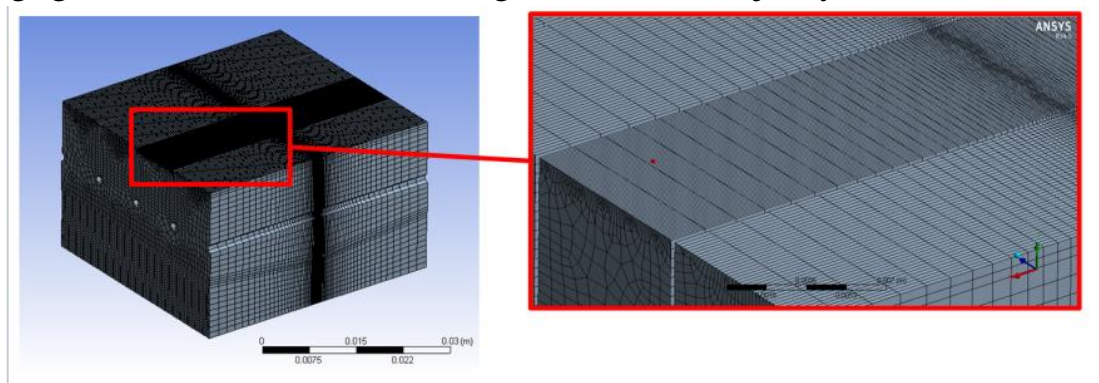

Figure 16: Solid mesh of the correlation model, showing heavy transverse refinement on all fins, and dense longitudinal refinement on fin 5. 


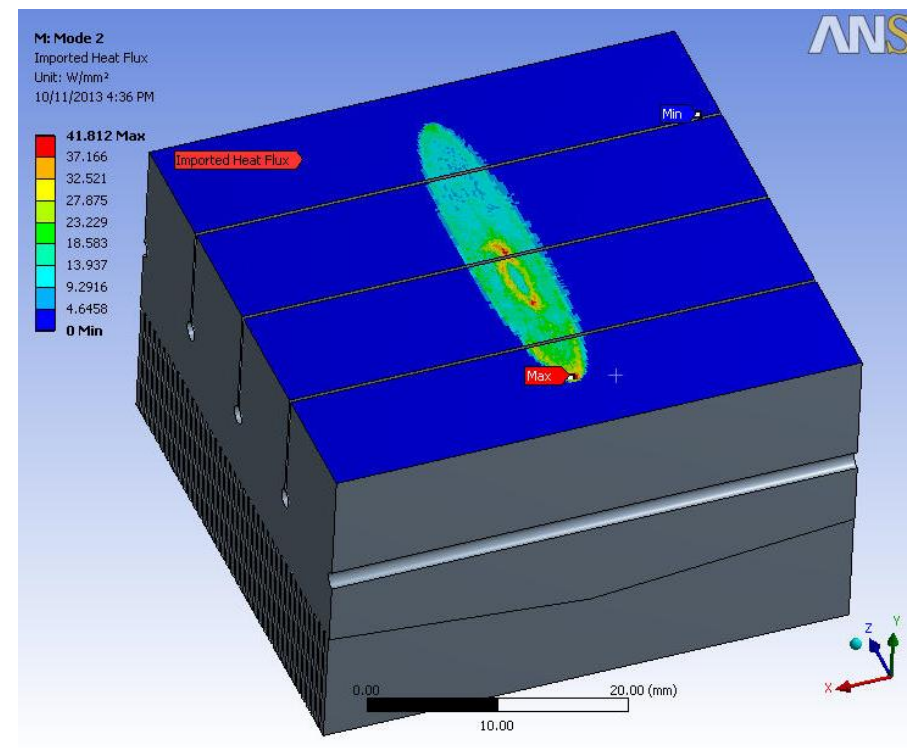

Figure 17: Underlying solid geometry of finite element model, with the peak-power-density test bench beam profile mapped as a surface heat flux load.

In conclusion, a prototype of a single absorber module was designed and built. TZM components were fabricated using a combination of EDM and conventional machining. The use of carbide cutting tools resulted in very limited and acceptable chipping in the inherently brittle TZM. In order to accomplish the required vacuum-tight water connections, processes for TZM-TZM braze joints and TZM-Titanium e-beam welds were developed. The prototype is not without warts, and required an expected amount of rework. Though challenging, the fabrication proved feasible, and could be scaled up to a four-module design. A finite element model was prepared and used to make thermal predictions. These analyses were then compared to and refined by prototype tests, which will be described in the following section.

\section{Test results}

Thermal properties of the absorber prototype were tested with an electron beam at a test stand described in Section 5.1. The main goals of these tests were to expose the absorber prototype to a power density comparable with what is required for PXIE; to verify simulations and assumptions on which the absorber design was made; and to gain experience of working with a high power density beam.

To make sure that the absorber mode of operation is representative, significant efforts were devoted to measure the parameters of the power deposition. It involved measuring the total deposited power (Section 5.2) and the current density distribution across the beam footprint (Section 5.3).

Surface temperature can be, in principle, reconstructed from the intensity of thermal radiation (Section 5.4). Uncertainty with calibration of the imaging system makes it difficult to use as a primary tool for the temperature analysis, but the intensity data is still useful for crosscorrelation and probably even more important, as a part of a protection system.

Information about the temperature distribution in the absorber prototype body came from a set of thermocouples (Section 5.5). In Section 5.6 it is correlated with simulations and optical measurements. Effects of the cooling liquid flow on the temperature distribution are presented in Section 5.7. 


\subsection{The test stand}

The test stand is described in [5], and its schematic is shown in Figure 18. A $27.5 \mathrm{keV}$, up to $0.2 \mathrm{~A}$ electron beam is generated in an electron gun, is focused by two solenoids, and either passes through a test chamber into an electrically insulated collector or is directed by dipole correctors to the surface of the prototype absorber. The port opposite to the absorber is covered by a quartz vacuum window for optical measurements, with an additional lead glass installed on the air side for radiation protection. Typical vacuum in the gun/test chamber is $0.1 / 3 \mathrm{nTorr}$ with the cathode off, and $\sim 10 / 70 \mathrm{nTorr}$ with the 0.2 A beam on the prototype.
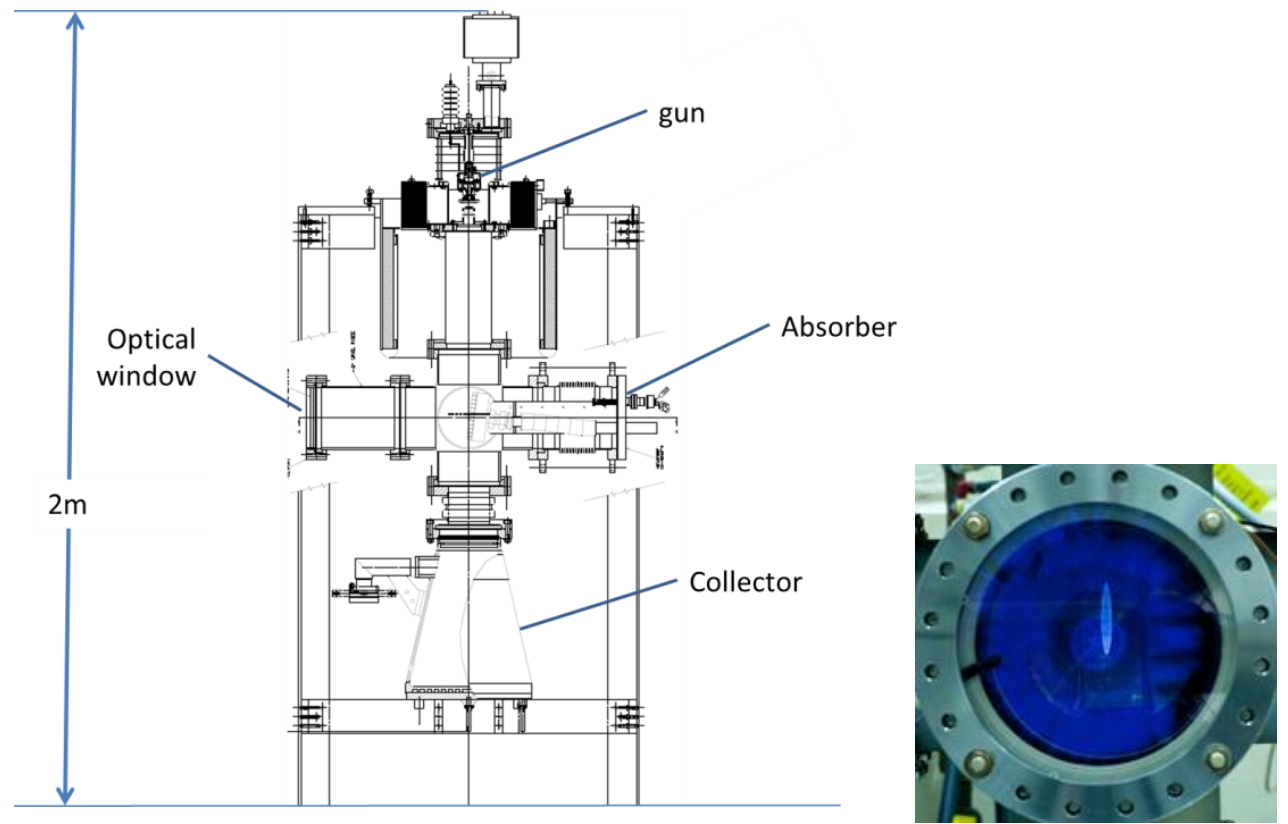

Figure 18: Absorber test stand. Left - schematic. Right - photo of the beam image (M. Murphy).

\subsection{Energy deposition}

Power of the electron beam is easily calculated from the electron energy $e U_{e}$ and the beam current $I_{e}$. The electron energy is determined by the cathode potential with respect to ground $U_{e}$, which was kept in all measurements equal to $27.5 \mathrm{kV}$. The beam current is equal to the bias power supply current minus the current of the resistive divider of $0.6 \mathrm{~mA}$. However, the figure of merit, the power dissipated in the absorber is greatly affected by energy taken away from reflected electrons and secondary particles. Below the portion of power removed from these processes is referred as a "power reflection coefficient."

An estimate of the power reflection coefficient was made using simulation by CASINO code [24]. However, at the shallow grazing angle, the results may depend on the surface properties, and a dedicated measurement is preferable. Such measurement was performed using the temperature difference between the input and output of the cooling circuit.

One of difficulties was an unknown thermal capacitance of the cooling liquid, composed of water and glycol in an unspecified ratio. The uncertainty was addressed in two ways. First, the thermal capacitance of the coolant $C_{\text {cool }}$ was compared with water's by measuring the heating curves of similar masses in the same vessel. The ratio was found to be 0.87 (volumetrically). 


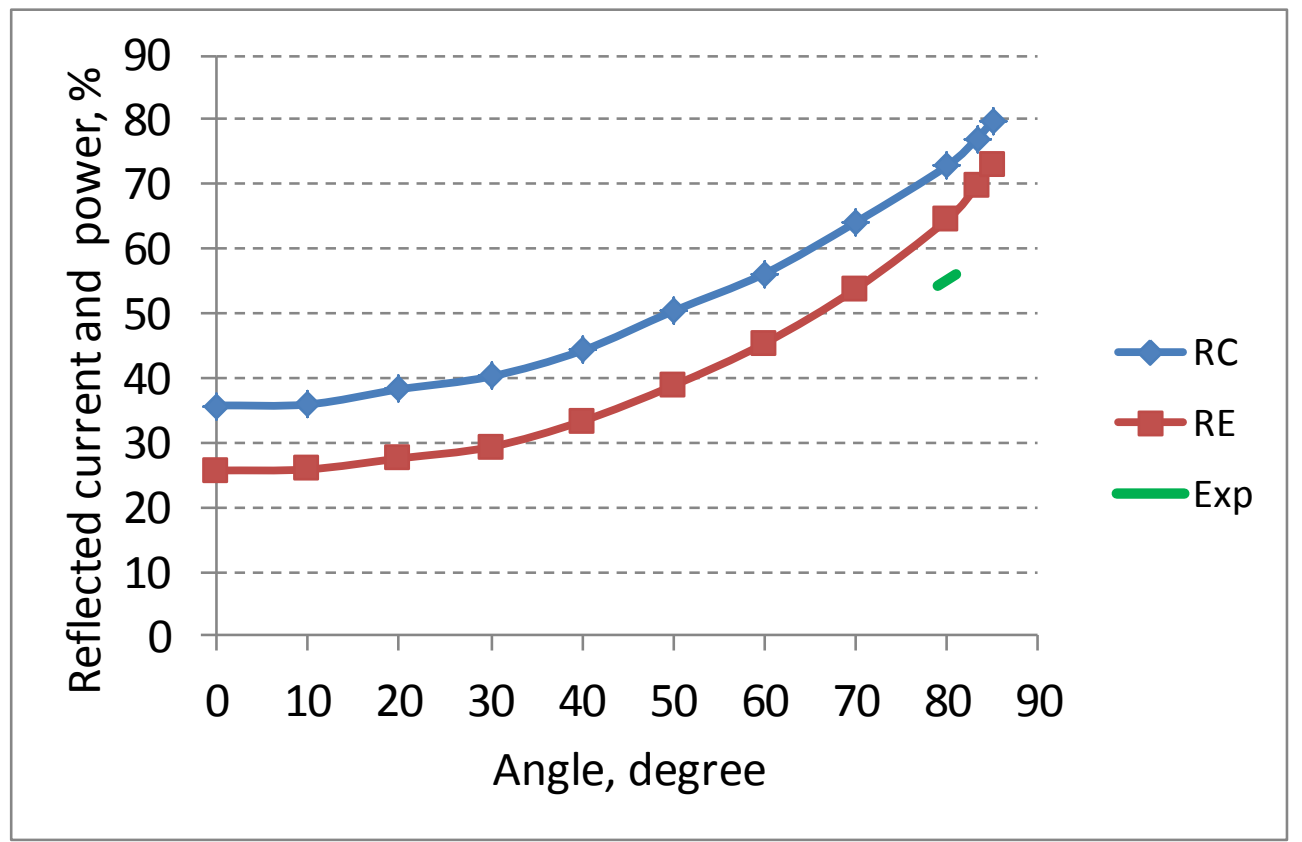

Figure 19: Portions of current and power of an electron beam reflected from a TZM surface as a function of the incident angle. Lines labeled $\mathrm{RC}$ and $\mathrm{RE}$ show result of simulation by the CASINO code, and the green line represent results of measurements of the energy not collected by the absorber prototype (see actual data in Figure 21). The horizontal axis is the grazing angle (i.e. 0 corresponds to a normal incidence).

Second, the cooling system with its measuring devices (a flow meter and thermocouples) was relocated to the collector of the test stand, and the beam was steered into the collector. According to both geometrical estimations and measurements, the collector confines $\sim 98 \%$ of the incoming beam current, so the power reflected from it is $<2 \%$. The input-to-output temperature rise $\Delta T_{i o}$ was measured in the range of the beam currents of $I_{e}=10-100 \mathrm{~mA}$ and coolant flows of $w_{c}=0.13-0.41$ liter/s. For each flow value, the temperature difference at zero current was subtracted to remove offsets and contribution of the heat exchange with air. If all beam power is removed by water and all measurements are perfect, the combination of $K_{c} \equiv \Delta T_{i o} \cdot w_{s} / I_{e}$ should be constant, $K_{c}=e U_{e} / C_{c o o l}$. While statistical errors of individual measurements were several percent, the measured values of $K_{c}$ systematically deviated from a constant as

$$
K_{c} / K_{c}\left(I_{e}=0\right)=\left(1-I_{e} / I_{0}\right) \cdot\left(1-w_{c} / w_{0}\right)
$$

with fitting coefficients $I_{0}=1400 \mathrm{~mA}$ and $w_{0}=2.4 \mathrm{l} / \mathrm{s}$. The first coefficient may be related to a heat loss into air through the upper part of the collector that could not be thermally isolated. The second coefficient is attributed to non-linearity of the flow meter (a $12 \mathrm{gpm}$ vortex flow meter CP4-M1T1C1W1, purchased from flowmeters.com); a similar behavior was observed when bypassing part of the flow parallel to the collector. The value of $K_{c}$ agrees with the thermal capacitance measurement at, for example, $I_{e}=50 \mathrm{~mA}, w_{c}=0.41 \mathrm{l} / \mathrm{s}$. Then, the cooling system was connected to the absorber prototype, and measurements were repeated with similar coolant flows. In this case, the temperature rise was linear with the beam current within the scatter and was $45 \%$ of the rise in the collector at the same flow. Example of the measurements is shown in 
Figure 20. We conclude that the power reflection coefficient is $55 \% \pm 4 \%$ for the beam at the center of the absorber prototype.

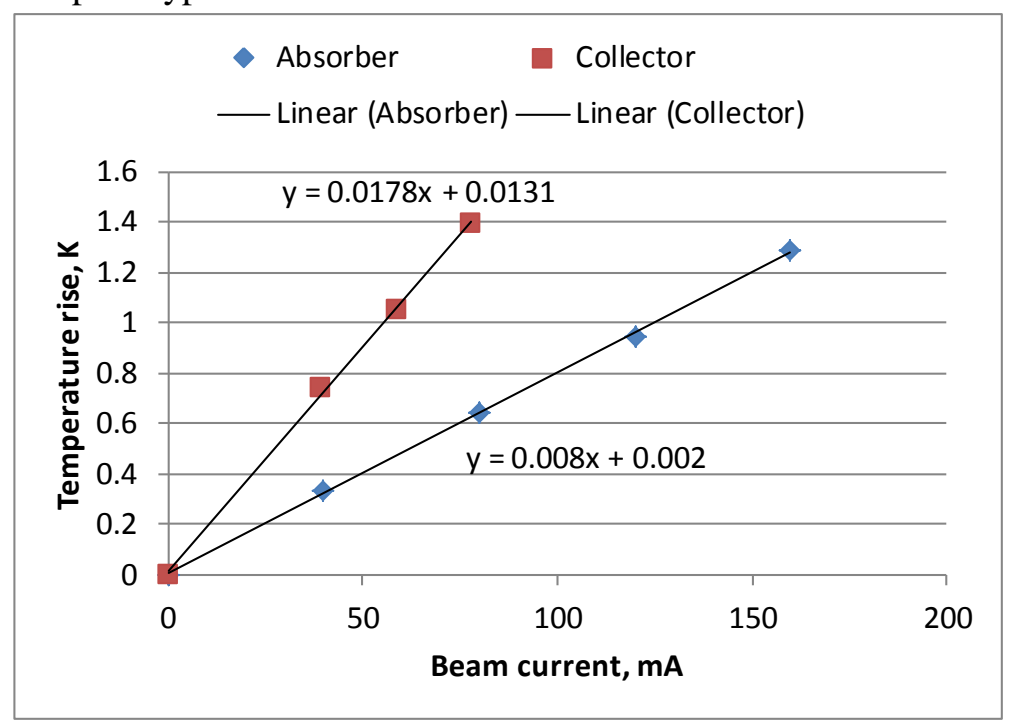

Figure 20 Temperature rise from coolant input to output as a function of the beam current in cases of the beam in the collector and at the absorber prototype. The coolant flow is $6.6 \mathrm{gpm}(0.41 \mathrm{l} / \mathrm{s})$.

The beam can be moved along the absorber surface with dipole correctors incorporated into the long solenoid. The move changes also the angle between the beam and the absorber surface. Correspondingly, the power reflection changes as well (Figure 21), though the range of the changes is within the systematic uncertainty.

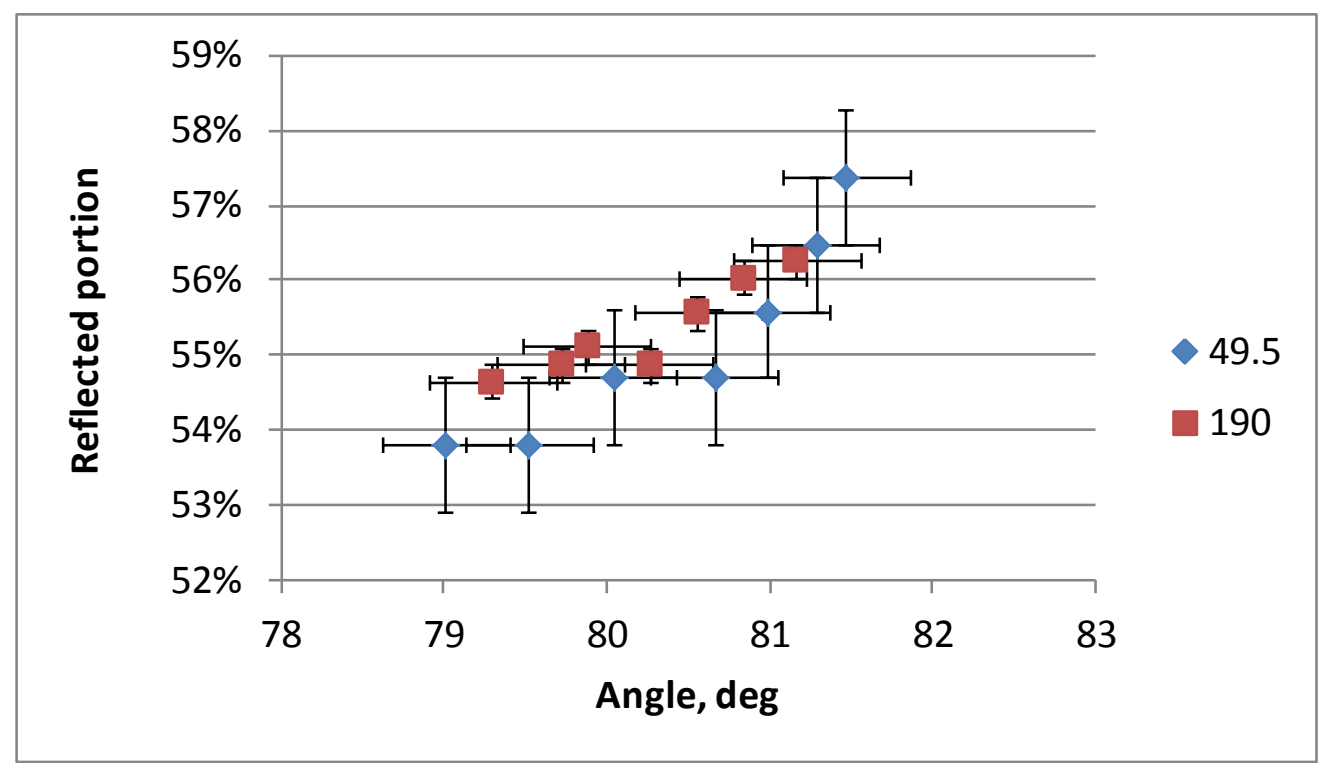

Figure 21: Power reflection coefficient as a function of the angle between the beam and the absorber surface. Two series correspond to different beam currents, 49.5 and $190 \mathrm{~mA}$. Vertical bars represent statistical error only. The systematic error, determined mainly by the calibration process described in the text, is $\sim 4 \%$. 


\subsection{Beam imaging and the beam footprint profile}

The surface of the absorber prototype installed at test stand can be viewed through a 6" quartz window and air-side lead glass shield by a CCD camera ${ }^{1}$ with resolution of 0.24 $\mathrm{mm} /$ pixel. The images can be captured and saved with a dedicated program ${ }^{2}$. Code Image J [25] was extensively used for visual presentation of images and preliminary analysis.

The image was dominated by two components, the Optical Transition Radiation (OTR) and the thermal radiation. The OTR is proportional to the beam current density and, therefore, provides information about the power density distribution. The thermal radiation characterizes the surface temperature.

Assuming that the reflection coefficient of TZM is nearly constant in the visible spectrum as it is for a pure molybdenum [26], the OTR has a flat spectral intensity distribution $d I / d v$. In contrast, the thermal radiation grows rapidly with increasing the wavelength. These properties were used to distinguish between two components. At low current density, the surface temperature is too low to produce significant thermal radiation in the visible spectrum, and the beam footprint image is determined by OTR. Therefore, in this regime the image intensity is proportional to the beam current. At high beam currents or small beam spot sizes, the surface temperature increases, and thermal radiation quickly becomes dominant. Without filtering, the threshold is typically at $600-700{ }^{\circ} \mathrm{C}$. However, the most interesting mode is exactly when the beam power density is high and, correspondingly, the surface temperature is well above this threshold. The thermal radiation contribution still can be suppressed by applying short-pass optical filters, though their application is limited by transmission of the lead glass. Parameters of the filters used in the measurements are summarized in Table 3. Also, Table 3 includes the lead glass transmission and relative sensitivity of the camera at filters central frequencies, which are shown for reference only and were not used in calculations.

Table 3. Parameters relevant to optical measurements. The "Filter color" column indicates how the filter is referred in the text. Width of the transmission curve is at $50 \%$ of peak transmission. Glass transmission and camera sensitivity are shown at the filter's central wavelength. The OTR intensity is normalized by the intensity measured with the red filter.

\begin{tabular}{|l|c|l|c|c|l|c|}
\hline $\begin{array}{l}\text { Filter } \\
\text { color }\end{array}$ & $\begin{array}{l}\text { Central } \\
\text { wavelength, } \\
\text { nm }\end{array}$ & $\begin{array}{l}\text { Width, } \\
\text { nm }\end{array}$ & $\begin{array}{l}\text { Peak } \\
\text { transmission }\end{array}$ & $\begin{array}{l}\text { Lead glass } \\
\text { transmission }\end{array}$ & $\begin{array}{l}\text { Relative } \\
\text { camera } \\
\text { sensitivity }\end{array}$ & $\begin{array}{l}\text { Relative } \\
\text { OTR } \\
\text { intensity }\end{array}$ \\
\hline Violet & 407.6 & 36.5 & 0.57 & 0.61 & 1 & 0.57 \\
\hline Blue & 451.8 & 87.4 & 0.704 & 0.83 & 0.9 & 5.12 \\
\hline Yellow & 605.5 & 35.2 & 0.567 & 0.88 & 0.5 & 1.58 \\
\hline Red & 707.3 & 37.6 & 0.613 & 0.88 & 0.3 & 1 \\
\hline
\end{tabular}

In addition, Table 3 includes the measured relative intensity of the OTR light (normalized by the red filter), which was calculated by comparing the light intensity from images at the same beam parameters (far from the thermal radiation threshold) with different filters. One can compare this sensitivity to a simple estimate, assuming $d I / d v=$ const, constant transmission of the quartz glass, and the numbers for the lead glass transmission and

\footnotetext{
${ }^{1}$ Monochrome Progressive Scan CCD TM-9701 from www.jai.com

${ }^{2}$ The program was written and supported by R. Thurman-Keup
} 
camera sensitivity from the Table 3 . The discrepancy with measurements is up to a factor of $\sim 10$. However, we did not proceed with attempts to resolve the discrepancy by additional calibrations.
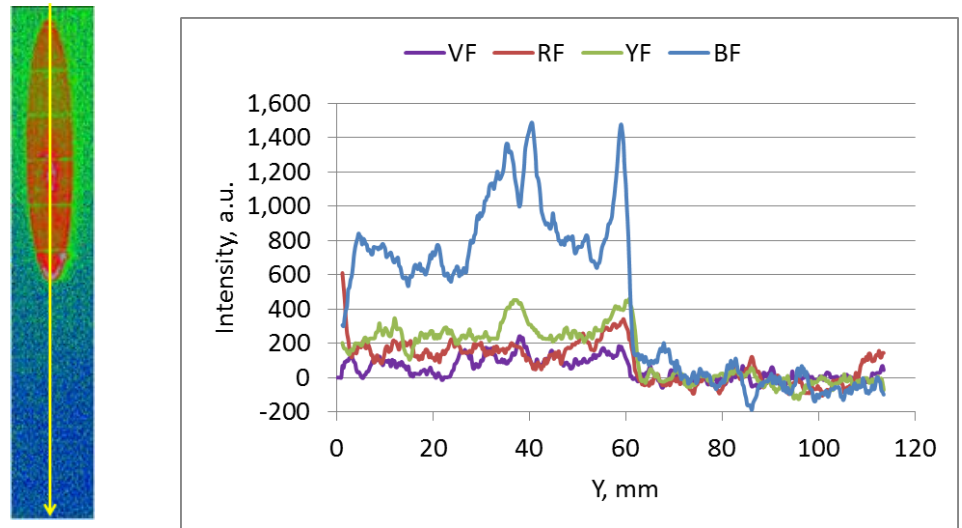

Figure 22. Sensitivity of the optical system to the OTR light. Left - one of beam images that were used for the calibration. The shown image was recorded with the blue filter and for presentation purpose is shown enhanced and in false colors. The horizontal lines correspond to the absorber slits with $10 \mathrm{~mm}$ spacing. A small bright spot in the lower right of the beam image is a feature of the surface and is interpreted as a thermal radiation from a micro particle (see "hot spots" in the text). Right- intensity distributions along the central line, represented by the yellow arrow on the left image, for four filters. The background is subtracted based on the signal outside of the beam footprint. The curves are shown with 10-point averaging. RMS value of the background fluctuations is $\mathbf{1 5 0}$ of intensity units used in the vertical axis.

The violet filter suppressed the thermal radiation signal below the noise level, but its OTR signal was found too weak to make a reasonable reconstruction of the beam profile. As a result, we had to use the blue filter for this purpose. However, with the blue filter the thermal radiation contribution became significant at the maximum tested power density. To overcome the difficulty, in this specific case the distribution was interpolated from the image of a slightly defocused beam, where the intensity integral was still linear with the beam current (see Section 5.6).

An unexpected observation was the existence of peculiar locations on the absorber surface that emitted a strong visible thermal radiation under the beam ("hot spots"). The hot spots started to emit at a very low power density, and typically did not change location within one session of measurements. Some hot spots were permanent, i.e. always present in the same location. Others seemed to move from day to day. In general, their population was decreasing over the months of testing. We were not able to associate the hot spots with any macroscopic feature on the absorber surface visible through the vacuum window (either with a bare eye or the camera). The lack of visible high-spots, the fact that some of the hot spots moved, and the observation that the number of hot spots decreased over time leads us to speculate that they might be dust particles, or some other form of weakly-adhered contamination. There were no indications that the hot spots affected performance of the absorber in any way (though sometime they did created inconvenience in optical measurements).

\subsection{Optical Temperature Measurements}

Intensity of thermal radiation contains information about the temperature of the radiating surface. In a case of the black body, the spectral radiance is, in $\mathrm{W} \cdot \mathrm{sr}^{-1} \cdot \mathrm{m}^{-3}$, 


$$
\frac{d^{3} I}{d \lambda d \Omega d S} \equiv L_{e \lambda}(\lambda, T)=\frac{2 h c^{2}}{\lambda^{5}} \frac{1}{e^{\frac{h c}{\lambda k_{B} T}}-1},
$$

where $T$ its absolute temperature, $k_{B}$ is the Boltzmann constant, $h$ is the Planck constant, and $c$ is the speed of light. The spectral radiance of a real surface differs by emissivity $\varepsilon(\lambda)$. Intensity of the thermal radiation recorded by a camera through a narrow-band filter $f(f=v, b, y, r$ for filters described in Table 3) is

$$
I_{c f}=C_{c}\left(\lambda_{f}\right) \frac{\varepsilon\left(\lambda_{f}\right)}{\lambda_{f}^{5}} \frac{\Delta \lambda_{f}}{e^{\frac{h c}{\lambda_{f} k_{B} T}}-1},
$$

where $\lambda_{f}$ and $\Delta \lambda_{f}$ are the central frequency and bandwidth of the filter, and the coefficient $C_{c}\left(\lambda_{f}\right)$ includes parameters of the camera and optical system. Measurements of the surface temperature employed mainly two filters, red and yellow (see Table 3). For both filters, the value of $T_{\text {eff }}\left(\lambda_{f}\right)=\frac{h c}{\lambda_{f} k_{B}}$ is much larger $\left(T_{\text {eff }}\left(\lambda_{r}\right)=20350 \mathrm{~K}\right)$ than the surface temperature $(<2000 \mathrm{~K})$, so the temperature can be calculated as

$$
T=T_{e f f}\left(\lambda_{f}\right) / \ln \frac{K_{f}}{I_{c f}}
$$

where $K_{f}=C_{c}\left(\lambda_{f}\right) \frac{\varepsilon\left(\lambda_{f}\right)}{\lambda_{f}{ }^{5}} \Delta \lambda_{f}$. The capability to visually observe the variation in temperature across the beam footprint in real time was very valuable in the tests. To quantitatively reconstruct the surface temperature distribution from measurements with a narrow-band filter, one needs to know the single parameter, $K_{f}$. Though uncertainties of $K_{f}$ contribute only logarithmically into the value of the temperature, many parameters of the optical system were not known with enough accuracy to calculate the value of the coefficient directly, and we made several attempts to determine $K_{f}$ by fitting to experimental data.

The first case was in tests of heating up a radiation-cooled TZM plate with an electron beam at the same test stand and using the same red filter [27]. While fitting of $K_{r}$ in these tests was dependent on unknown values of the beam power reflection coefficient and emissivity, with reasonable assumptions the corresponding uncertainty in temperature of the absorber surface became $\pm 100 \mathrm{~K}\left(K_{r}=2 \cdot 10^{11}-2 \cdot 10^{12}\right)$.

Another possibility was to fit simultaneously measurements with red and yellow filters, using Eq. (6) and the following assumptions:

1. The beam image is reproducible when filters are changed. Note that filter changing was done manually and required shutting down the beam.

2. The image of the defocused beam recorded with the blue filter is not affected by the thermal radiation and, therefore, can be used to subtract the OTR component from red and yellow filter images.

3. Emissivity is the same for both red and yellow wavelengths.

4. The spectral (frequency) density of OTR radiation in measurements described in Section 5.3 is the same for both filters, so the OTR can be used for cross-calibration. Then, the measured ratio of the camera signals with red and yellow filters (see Table 3) is 


$$
R_{y r}=1.58=\frac{C_{c}\left(\lambda_{y}\right)}{C_{c}\left(\lambda_{r}\right)} \frac{\Delta \lambda_{y}}{\Delta \lambda_{r}} \frac{\lambda_{r}^{2}}{\lambda_{y}^{2}},
$$

where indexes indicate corresponding filters. It gives a relationship between coefficients $K_{y}$ and $K_{r}$ as

$$
K_{y}=K_{r} \cdot R_{y r} \cdot\left(\frac{\lambda_{r}}{\lambda_{y}}\right)^{2}
$$

Therefore, one can try to adjust $K_{r}$ looking at the best agreement between temperature curves reconstructed with Eq. (7) from the images recorded with the yellow and red filters. For this calibration, images of the beam at the same parameters were recorded with three filters. The beam focusing was chosen for the power density to be right below the threshold of appearance of the thermal emission signal with the blue filter. Then the OTR component was subtracted from the red and yellow images using coefficients found in measurements with a defocused beam (Table 3). Temperature profiles calculated with Eq. (7)-(9) for both cases were compared at various values of the coefficient $K_{r}$. One of such results is shown in Figure 23. There is significant scatter in the best fitting values of $K_{r}$ depending on what portion of the image is chosen for fitting. Corresponding uncertainty for the temperature is $\pm 150 \mathrm{~K}$ ( $K_{r}=2 \cdot 10^{11}$ $\left.2 \cdot 10^{12}\right)$.
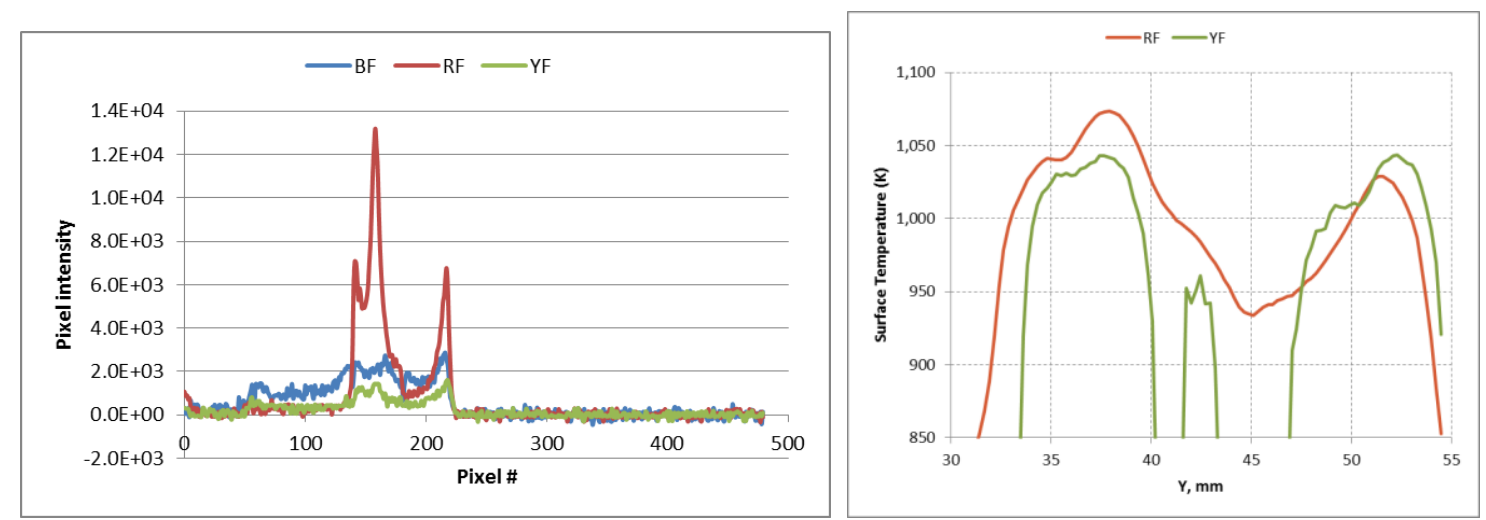

Figure 23. Temperature reconstruction from filtered images. Left: distribution of the light intensity recorded along the central line with three filters. $I_{e}=190 \mathrm{~mA}$. Background subtracted. Right: example of temperature reconstruction from data on the left. The signals are shown with 10-point averaging. $K_{r}=1.6 \cdot 10^{12}$.

Additional restriction comes from comparison with thermocouple measurements discussed in Section 5.5. Note that measurements at the maximum power density were performed with a red filter in combination with two neutral filters of $10 \%$ and $1 \%$ transparency.

\subsection{Thermocouple Temperature Measurements}

As described in 4.2, the absorber was instrumented with six Type-K thermocouples. Fin 5 was instrumented with four of the six thermocouples; this location was subjected to the peak of 
the energy deposition for correlation purposes. The locations of the thermocouple beads are are shown in Table 4.

Table 4: Thermocouple Location Information

\begin{tabular}{|c|c|c|c|}
\hline ID & Fin \# & $\begin{array}{c}\text { Depth from Surface } \\
(\mathrm{mm})\end{array}$ & $\begin{array}{c}\text { Transverse Offset } \\
(\mathrm{mm})\end{array}$ \\
\hline TC01 & 5 & 2.65 & 0 \\
\hline TC02 & 8 & 2.42 & 0 \\
\hline TC05 & 5 & 33.15 (below coolant) & 0 \\
\hline TC06 & 5 & 6.15 & 6 \\
\hline TC07 & 5 & 9.15 & 0 \\
\hline TC08 & 9 & 6.15 & 8 \\
\hline
\end{tabular}

It was desired to measure internal (rather than surface) temperatures at several locations within the absorber body, and to do so without introducing large holes that would substantially affect the thermal conduction paths. In order to do this, small thermocouples were selected. These thermocouples contained an ungrounded junction in an Inconel sheath with 0.032 " diameter. Long holes were drilled from the upward (gun facing) and downward (collector facing) faces of the absorber. The holes were $50 \mu \mathrm{m}$ larger than the thermocouple sheath. The sheath was inserted into the hole until the thermocouple reached the desired location. A slight kink was placed in the sheath to retain it in the desired position by friction.

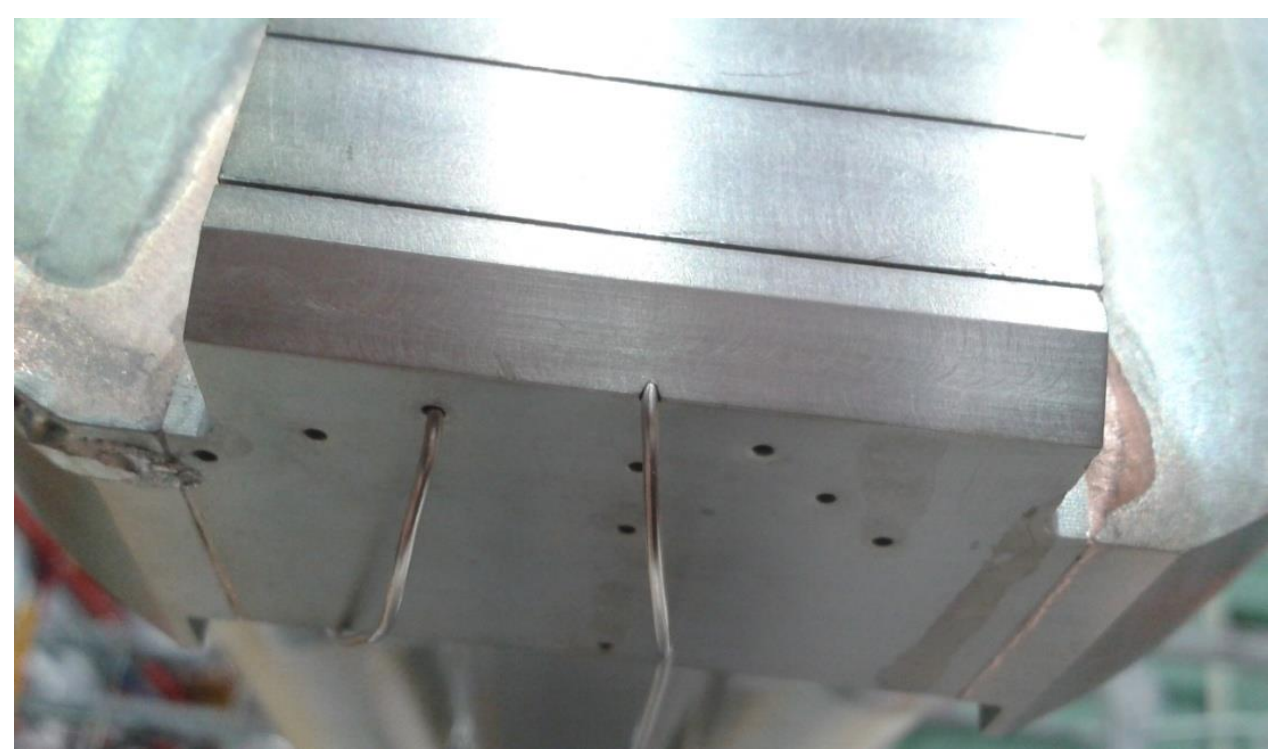

Figure 24. Two thermocouple cables entering the absorber body at the downward (collector-facing) face.

Due to the inability to provide direct clamping between the thermocouple bead and the absorber body, there was poor thermal contact. For this reason, conduction along the thermocouple could relatively easily influence the reported temperature. This was observed when scans were taken with a small "pencil beam," with a size substantially smaller than the $10 \mathrm{~mm}$ fin pitch. When the fin containing a thermocouple bead was irradiated, the thermocouple would respond. When the irradiated the adjacent fin through which the sheath passed, the 
thermocouple showed a response, generally about $20 \%$ as high as the peak when the beam was centered on the bead. If the beam irradiated the adjacent fin on the opposite side of the sheath, there was no thermocouple response. In this sense, the thermocouple is measuring a weightedspatial-average temperature of two fins. In cases where beam was small and thermal loading varied significantly between the bead fin (fin 5) and the sheath fin (fin 4), this affect was accounted for during correlation.
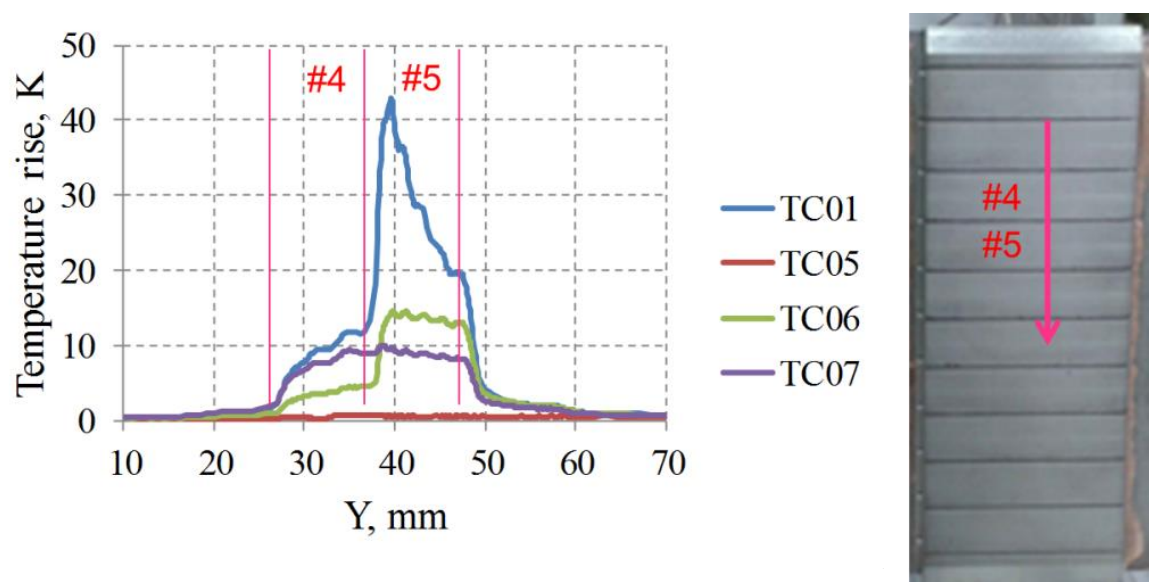

Figure 25: Temperature results when a small beam is scanned from top to bottom. Response starts when beam is in fin 4 due to conduction along the thermocouple sheath. Response peaks in fin 5 when the beam is over the bead. As the beam moves onto fin 6, temperatures decay, with little or no conduction effect observed between fins 5 and 6 .

\subsection{Analysis of test and correlation results}

Correlation between analysis predictions and thermocouple measurements was attempted for several beam conditions. These efforts resulted in tracking down and minimizing measurement uncertainties (e.g. the actual thermal properties of the coolant as mentioned in Section 5.2), and modeling uncertainties as described in Section 4.4. At the end of these efforts, comparison was made between the prediction and thermocouple measurement in two focusing conditions (identical to those used for optical surface temperature measurement).

In the intermediate-focusing case, the beam profile was reconstructed from blue-filtered OTR as described in Section 5.3, and mapped to the ANSYS model described in Section 4.4. The results of mapping are shown in Figure 26.

The tight-focusing case produces the peak PXIE-requirement surface power density of $17 \mathrm{~W} / \mathrm{mm}^{2}$ (spatial average over fin 5). However, at this focusing significant thermal radiation has a wavelength short enough to pass the blue filter. As such, blue-filtered results could not be reliably used to reconstruct the beam profile. In order to overcome this and generate a profile for use in analysis, the beam profile from the intermediate focusing case was scaled to have the known overall size as the tight-focusing case. Total beam power was invariant, so power density increased. Scaling the intermediate-focusing profile resulted in a profile with 17 $\mathrm{W} / \mathrm{mm}^{2}$ average, and $40 \mathrm{~W} / \mathrm{mm}^{2}$ peak. This was used as an input to analysis. 


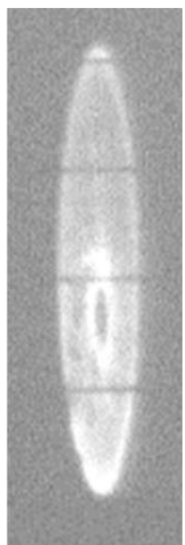

Blue-filter beam image Dominated by OTR

\section{Mapping to FEA}

OTR intensity

proportional to

power

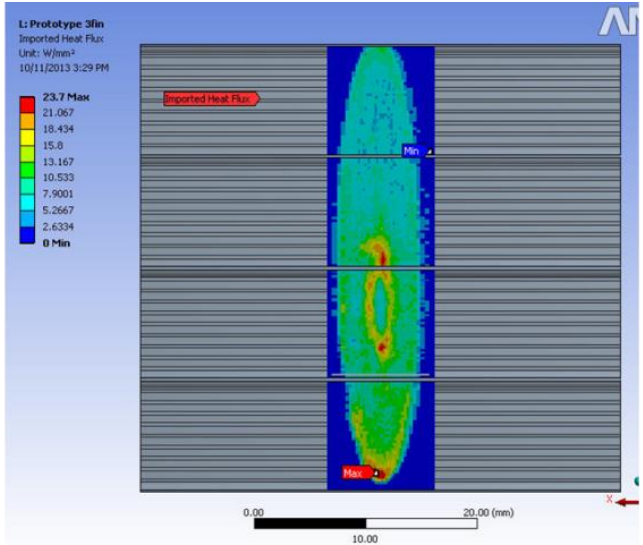

Analyzed beam profile: intermediate focusing

Power Density on Fin 5"

$10 \mathrm{~W} / \mathrm{mm}^{2}$ average $25 \mathrm{~W} / \mathrm{mm}^{2}$ peak

Figure 26: "Intermediate focusing case" beam profile as mapped onto the finite element model. OTR intensity is proportional to the current density.

Analysis predictions are compared to measurement results in the table below. For measurements of surface temperature, analysis is compared to the optical measurements described in Section 5.4. For all bulk temperatures, analysis is compared to thermocouple measurements. In the area of peak surface temperatures, and at the areas of discrete thermocouple measurements within the bulk, measurements and predictions agree to within about $10 \%$. Agreement between analysis and optical measurements is worse away from the peak of the beam profile.

Table 5: Analysis Correlation Results

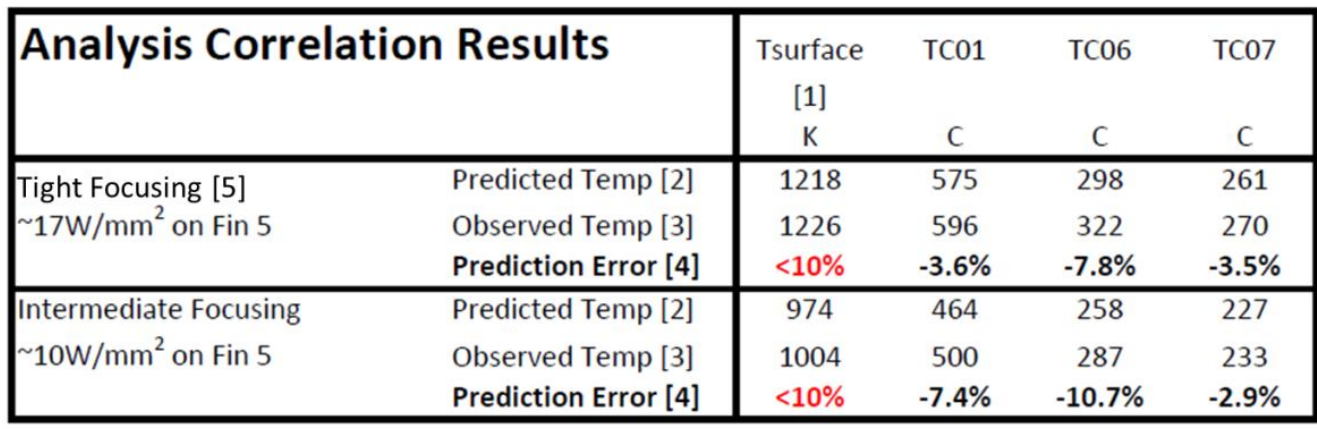

Notes:

[1] Average temperature in longitudinal trace over center of fin 5 Errors of order $20 \%$ in the cooler areas of the surface

[2] Predicted in FEA, with thermocouple conduction effect compensated

[3] Surface observation from optical measurement, all others from thermocouples

[4] calculated as [dT predicted - dT observed] / [dT observed] where $\mathrm{dT}$ is change relative to water temperature

[5] Beam profile is a scaling of the OTR-dominated intermediate focusing profile 

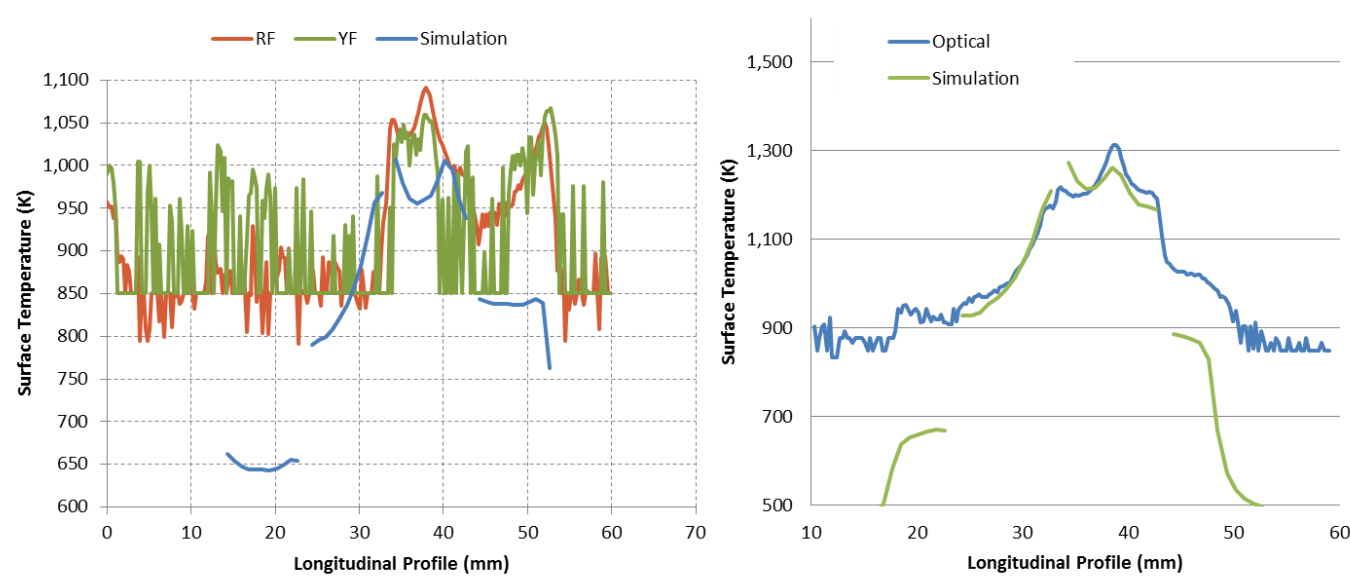

Figure 27. Example of agreement between analysis and optical surface temperature measurement in two focusing conditions. Left: intermediate focusing. Right: Tight focusing

Agreement between the model prediction and thermocouple measurements is not enforced. That is, no tweaks or changes were made to the model to accomplish the agreement shown. Agreement between the model prediction and the optical measurement IS enforced; the values chosen for the parameter $K_{f}$ (see Eq.(7) in Section 5.4) was allowed to vary within the limits of its uncertainty to optimize agreement between optical temperature measurement and analysis prediction.

\subsection{Cooling Studies}

As described in Section 3.2.4, the cooling channels were expected to provide laminar flow across the expected range of flow rates. For truly laminar flow, the convection coefficient $h$ does not depend on flow rate: one should be able to increase or decrease flow without affecting the heat transfer characteristics. In contrast, the heat transfer characteristics in the turbulent or transition flow regimes do change with flow rate - higher flow rates provide more turbulence and better heat transfer.

In order to characterize the prevailing flow regime, a series of tests was performed. Stable beam conditions were established, providing PXIE-like $17 \mathrm{~W} / \mathrm{mm}^{2}$ peak power density. Flow rate was incrementally changed from a low value of $2 \mathrm{gpm}$ (which provides a peak fluid velocity of approximately $0.6 \mathrm{~m} / \mathrm{s}$ in the cooling channels) up to high value of $12 \mathrm{gpm}$ (peak fluid velocity $3.5 \mathrm{~m} / \mathrm{s}$ ). Thermocouples were monitored for any inflections that would indicate a change in heat transfer regime.

An example of the test data is shown in Figure 28. Reading of the thermocouple TC01, which measures the high temperatures near the absorber surface, decreased only slightly as flow was increased.

From a first-order inspection of the data, it is clear that changing the flow rate has no strong effect on absorber temperatures. In order to look for more subtle effects, the changes in absorber thermocouple measurements were compared to changes in outlet water temperature. For constant power being carried away in the coolant, as the flow rate decreases the temperature rise of the coolant must increase. A simplistic, but adequate, assumption is that the absorber is cooled by flow with an effective temperature that is the average of inlet and outlet temperatures:

$$
T_{w_{-} \text {eff }}=\frac{T_{w_{-} \text {out }}+T_{w_{-} \text {in }}}{2}
$$




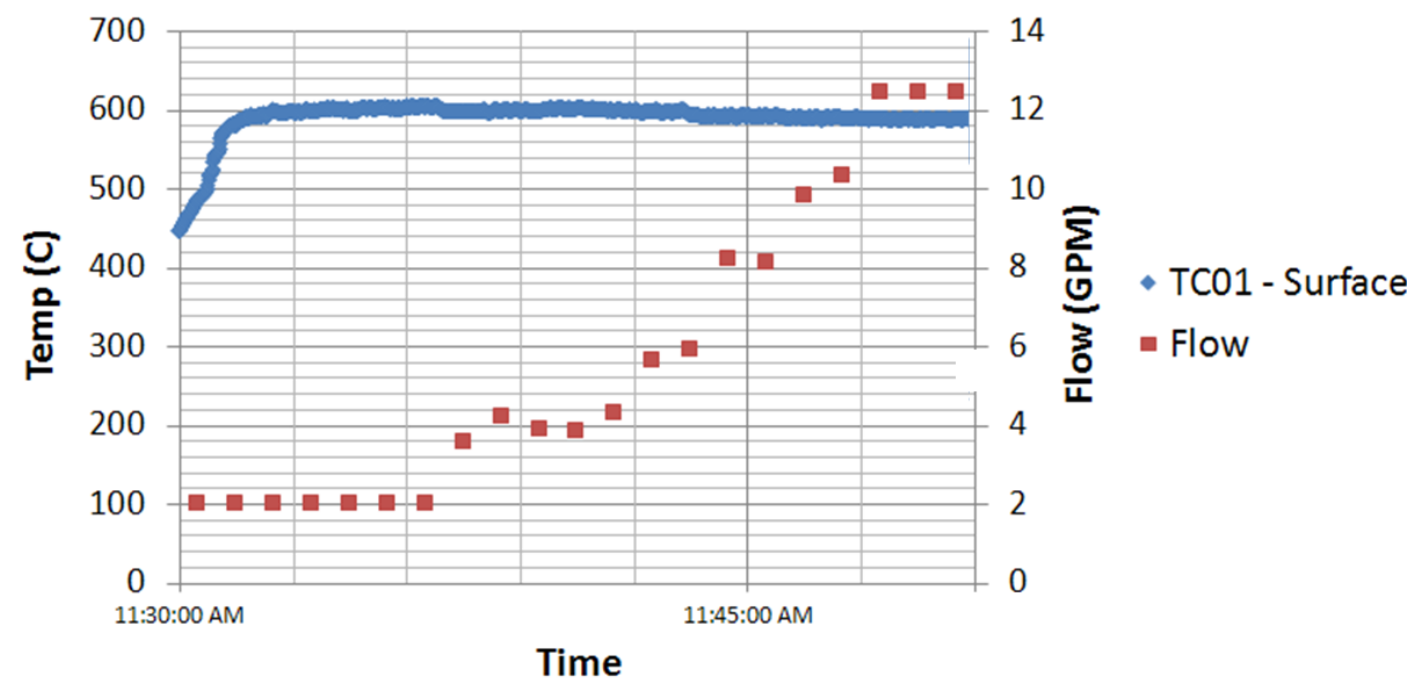

Figure 28. Example data - response of TC01 to flow changes under stable beam conditions.

The inlet and outlet temperatures are measured using the whole flow; no individual channel measurements are available. Extending the well-supported assumption that longitudinal conduction is not significant, it is assumed that the outlet temperature of each of the 10 cooling channels in a given fin has temperature rise proportional to the beam power in the fin:

$$
d T_{\text {fin } N}=T_{\text {out fin } N}-T_{\text {in fin } N}=\frac{P_{\text {fin } N}}{\rho \cdot c_{f} \cdot F_{\text {fin } N}}
$$

where

$T_{\text {out fin } N}$ is the average temperature across the channel outlets in fin $\mathrm{N}$

$T_{\text {in fin } N}$ is the inlet temperature, assumed to be uniform

$P_{\text {fin } N}$ is the power absorbed in fin $\mathrm{N}$

$\rho$ is the density of the fluid

$c_{f}$ is the heat capacity of the fluid

$F_{\text {fin } N}$ is the volumetric flow rate through the channels of fin $\mathrm{N}$. The proportion of the whole flow passing through fin $\mathrm{N}$ is estimated by fluid analysis

Equations (10) and (11) can be combined to calculate the effective cooling temperature in a given fin:

$$
T_{\text {eff_f finN }}=\frac{T_{w_{-} i n}+\left(T_{w_{-} i n}+d T_{f i n N}\right)}{2}=T_{\text {in fin N }}+\frac{P_{f i n N}}{2 \rho \cdot c_{f} \cdot F_{f i n N}}
$$

So as flow is increased, one would expect the effective cooling temperature to decrease. In the absence of any changes in cooling regime, one would expect all thermocouples in fin 5 to track the effective cooling temperature for fin 5 degree-for-degree.

When the thermocouple temperature data were compared to the effective cooling temperature of fin 5, it was found that, with increasing flow, thermocouple temperatures fell more than could be explained by the effect described in Eq. (12). This is shown in Figure 29. It appears that a roll-off begins at a flow between 4 and $6 \mathrm{gpm}$; and temperatures are slightly lower than one would expect if flow remained purely laminar. This may indicate that the onset 
of the transition to turbulent flow. This onset could be aided by the relatively short length of the channels and the sharp geometric discontinuities at the channel inlet and outlet locations.

Though beneficial, this effect is still small: lowering of temperatures relative to the laminar expectation is $<10^{\circ} \mathrm{C}$. The primary conclusion is that the design of the cooling channels is not very sensitive to flow rate, and that low flows can be accommodated with very modest effects on temperature. There is no strong indication of boiling or any other effect which could lead to abrupt changes in the cooling performance.

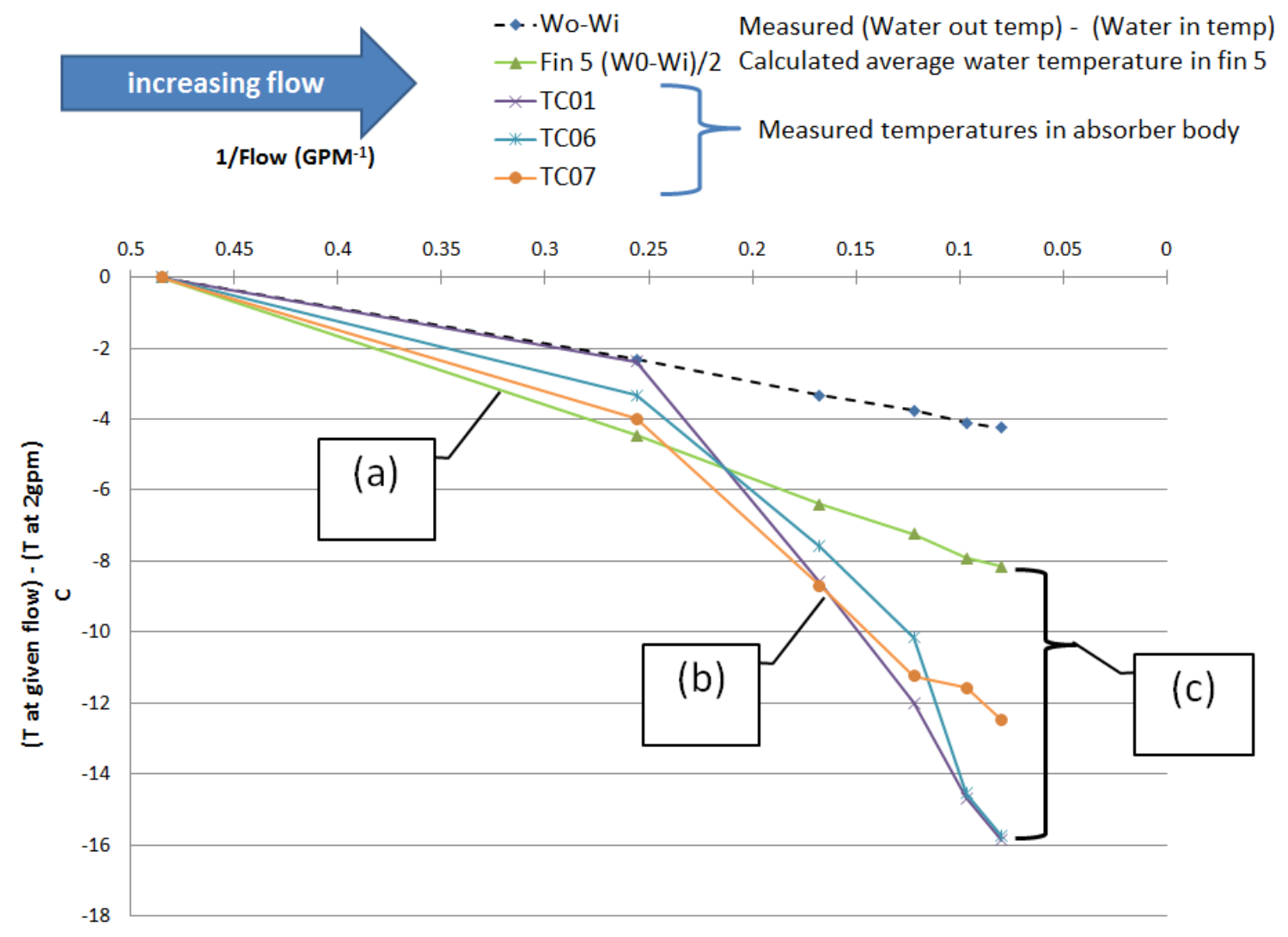

Figure 29. Flow test processed data - response of thermocouples to increasing flow rates. Figure notes:

(a) For laminar flow with constant performance, all measured temperatures should coincide with this line.

(b) Instead, the thermocouple curves roll over at higher flow. Lower-than-expected body temperatures indicate that convection coefficient " $h$ " may be increasing with flow. This may be an indication of turbulent onset.

(c) Roll-off of $8^{\circ} \mathrm{C}$ max as compared to what we would expect with invariant cooling.

In conclusion, the absorber prototype was tested on a dedicated electron-beam test stand. Beam footprint was measured optically via OTR. Temperatures were measured optically via visible thermal radiation and physically via thermocouples. Measured temperatures were correlated with analysis predictions with reasonable $(\sim 10 \%)$ agreement. Over a several-month test program, the test bench power and focusing were increased until peak absorbed power density replicated the $17 \mathrm{~W} / \mathrm{mm}^{2}$ associated with a $10 \mathrm{~mA}$ PXIE beam. The absorber survived without apparent damage, suggesting that the design is adequate. No thermal cycling was 
performed due to the concern that a failure would compromise our ability to test alternate design concepts. More detailed conclusions are listed and organized in the next, final section

\section{Conclusions}

The primary result of this testing program is that a prototype was built, tested under representative thermal conditions, and survived. Conclusions will be separated into two sections: a summary of detailed lessons learned (6.1), and a final section of broad conclusions (6.2).

\subsection{Lessons Learned}

Manufacturing

- A risk was taken in using lower-cost TZM 364 (powder metallurgy) rather than 363 (vacuum arc melted). Though the material was brittle, it withstood the fabrication and testing. There was no indication of unacceptable porosity.

- In addition to the Electro-Discharge Machining that is standard practice for TZM, the material was successfully machined with carbide cutting tools. There was a small and acceptable amount of edge chipping.

- TZM-to-Titanium E-beam welding was workable. There were some visual indications of weld embrittlement, but the welds did not functionally exhibit more brittleness than the TZM itself.

- Palcusil 25 braze alloy wet TZM well, as suggested by literature. Au35/Cu65 (applied as a paste and brazed in $\mathrm{H}_{2}$ ) produced a workable joint, but exhibited inferior wetting.

- Brittle fractures in some test pieces suggest a need for better surface preparation. A possible approach is incremental grinding with progressively smaller abrasive grains.

- Electropolishing was not very effective at removing TZM material or smoothing surface features, and left a tenacious blue residue in some of the small features. Process development would be required if electropolishing is needed to remove surface flaws.

- In-process QA leak checks proved their worth by catching a leak through the TZM bulk early in the fabrication process. Rigorous QA would be required for a 4module PXIE build.

Diagnostics and Instrumentation

- Measurement of OTR proved indispensable in understanding the beam profile. Unfortunately, calculations indicate that OTR produced by $2.1 \mathrm{MeV} \mathrm{H}-$ in PXIE will be undetectable.

- Measurement of thermal radiation is critical, and may be the most useful tool for setting up protection of the absorber. Thermal radiation should be easily detectable in PXIE.

- Measurement of surface temperature via thermal radiation would have benefitted from an unambiguous calibration to reduce uncertainty. 
- Thermocouple performance was compromised by poor thermal contact at the bead and significant conduction along the sheath. In future designs, means may be provided for more positive contact at the bead.

- The insertion of thermocouples through long longitudinal holes was troublesome; dimensional variations in the thermocouples made some of the procured thermocouples unusable. A different geometry with transverse routing would be preferable.

Vacuum

- Initial irradiation of the surface produced significant outgassing. As expected, burn-in of the PXIE absorber will be required to achieve stable and acceptable vacuum.

- Once equilibrium was reached, beam-on vacuum was stable at or below $10^{-7}$ Torr, likely determined by by outgassing caused by secondary particles. This is compatible with the expected PXIE vacuum in the absorber region in the mid $10^{-7}$ Torr range.

Cooling

- An unknown mixture of water and glycol(s) was present in the test bench; significant effort had to be expended to understand the properties of this mixture.

- The understanding that $\sim 25 \%$ of $\mathrm{H}$ - energy will be reflected from the primary absorbing surface in PXIE moves the cooling into a more comfortable regime. As such, water at $20^{\circ} \mathrm{C}$ would work for PXIE, rather than more aggressive solutions initially imagined.

- Cooling performance is stable at single-module flow rates between 2 and 12 gpm, suggesting that laminar flow dominates. Given that low flow rates seem to be adequate, this provides additional design space for the PXIE design.

- Between 2 and $12 \mathrm{gpm}$, there was no indication of strong changes in flow or cooling regime. There is slight indication of turbulent onset and associated improved cooling above $4 \mathrm{gpm}$.

Analysis

- Incorporation of the actual structure of the test bench beam profile was critical to producing results representative of the test.

\section{Miscellaneous}

- Some small areas of the surface (diameter $\leq \sim 1 \mathrm{~mm}$ ) emitted strong thermal radiation, even at low power density. These "hot spots" gradually decreased over time, it is speculated that they may be the result of dust on the surface. Apart from inconvenience in optical measurements, the hot spots did not appear to affect the performance of the absorber.

- Some subtly white areas were created on the absorber surface during testing. At least some of these changes occurred early on and at a low power density. It is speculated that this could be related to surface contamination.

- A crack-type failure of the prototype would have allowed glycol into the vacuum system, essentially destroying the test bench. Because of this fear, planned 
thermal cycles were not performed. Consequences of this sort of failure in PXIE would be dire, and consideration should be given to eliminating this failure mode.

\subsection{Summary and Top-Level Conclusions}

The primary conclusions of this testing program are as follows:

A prototype of the PXIE MEBT beam absorber has been built, and tested using an electron beam to an average absorbed power density of $17 \mathrm{~W} / \mathrm{mm}^{2}$. This is representative of PXIE operating at the full $10 \mathrm{~mA}$ beam current. The prototype survived the testing.

A multi-step fabrication process of the prototype absorber module proved challenging but doable. With rigorous QA, this fabrication approach could be extended to a four-module PXIE design.

Temperature measurements and finite element analyses agree to within the measurement uncertainty. This suggests that design and analysis methods were adequate, and can be extended to improve the design in future design iterations.

Testing interrogated several design details, with favorable results:

- Molybdenum alloy TZM withstood high temperatures of $\sim 1200 \mathrm{~K}$ as expected. TZM still appears to be the most appropriate material for this application.

- Vacuum performance of the prototype was adequate, with beam-on vacuum typically better than $10^{-7}$ Torr (after initial outgassing). Vacuum levels near the PXIE absorber are expected to be in the mid E-7 Torr range. This suggests that materials and construction techniques were adequate.

- Cooling performed as expected. The design operates far from any abrupt changes in cooling regime.

The testing program pointed out weaknesses of the current design that bear consideration in future design iterations:

- Better capture and management of reflected energy is desirable.

- The joints and material transitions required to have water flowing through the TZM material proved challenging to implement.

- A potential consequence of a crack through the TZM would be a coolant-intovacuum failure. This would have catastrophic consequences.

As of this writing, the prototype absorber is planned to be used as a temporary beam stop and chopper absorber during the integration and commissioning of the PXIE MEBT.

\section{Acknowledgments}

Fermilab is operated by Fermi Research Alliance, LLC, under Contract DE-AC02-07CH11359 with the U.S. DOE.

The authors acknowledge the direct technical contributions of the following, and offer apologies to any who may have been inadvertently omitted: 
K. Carlson (Test stand electrical and electronics engineering)

A. Chen (Absorber vacuum design)

A. Denisov (Thermal radiation measurement and analysis of the TZM plate)

Y. Eidelman (Beam interaction simulation and sputtering estimates)

C. Exline (Prototype assembly)

B. Hanna (Test stand, operation and measurements)

M. Hassan (Progenitor design concept)

A. Lumpkin and V. Scarpine (Optical filters)

V. Lebedev (Progenitor design concept)

A. Mitskovets (Test stand conditioning)

L. Prost (Test stand commissioning and simulation)

R. Thurman-Keup (Optical instrumentation)

J. Walton (Test stand, absorber pre-prototype)

The authors acknowledge the advice of:

V. Dudnikov, who pointed out the significance of the blistering effect,

T. Schenkel, who pointed out that H- reflected power would be large,

I. Terechkine, who suggested the micro-channel cooling implementation.

\section{References}

[1] S. Holmes et al., Proton Improvement Plan-II, December 2013, White paper

[2] S. Holmes et al., Project X reference design report, 2013, Project X document 776; S. Holmes, S. Nagaitsev, R. Tschirhart, Project X: a flexible high power proton facility, in proceedings of The APS Division of Particles and Fields Community Summer Study (Snowmass), July 29-August 6 2013, Minneapolis MN USA, SNOW13-00019, [physics.acc-ph/1305.3809]

[3] S. Nagaitsev et al, PXIE design handbook, 2013, Project X document 1148

[4] A. Shemyakin et al., Design of MEBT for the Project X Injector Experiment at Fermilab, in proceedings of Linear Accelerator Conference LINAC'12, Tel-Aviv, Israel, Sep. 9-14, 2012, MOPB095

[5] A. Shemyakin et al., Status of PXIE MEBT absorber development, in Proceedings of the 2013International Particle Accelerator Conference, May 12-17, 2013, Shanghai, China THPFI085

[6] A. Alexandrov, Overview and future demands of fast choppers, in Proceedings of Linear Accelerator Conference LINAC'10, September 12-17, 2010, Tsukuba, Japan, WE104

[7] F. Gerigk, M. Vretenar et al, Linac4 Technical Design Report, $11^{\text {th }}$ December 2006, CERN-AB2006-084 ABP/RF, p.22

[8] L. Bruno, M. Magistris and M. Silari, Conceptual design and radiological issues of a dump for the $3 \mathrm{MeV}$ test facility, $9^{\text {th }}$ February 2004, CERN-SC-2004-008-RP-TN

[9] H.D. Falter, E. Thompson, Performance of hypervapotron beam stopping elements in JET, JET$\mathrm{P}(95) 13$

[10] D. Oshatz et al., Mechanical design of the SNS MEBT, Proceedings of the 2001 Particle Accelerator Conference, June 18-22 2001, Chicago IL USA, TPAH152 
[11] S. Virostek, D. Oshatz, J. Staples, A Thermal analysis model for high power density beam stops, in Proceedings of the 2001 Particle Accelerator Conference, June 18-22 2001, Chicago IL USA, TPAH153

[12] T. Van Hagan, D. Doll and J. Schneider, Design of an ogive-shaped beamstop, in proceedings of Linear Accelerator Conference LINAC'98, August 23-28, Chicago, IL USA, TU4092

[13] V. Lebedev et al., PXIE optics and layout, in Proceedings of the 2012 Particle Accelerator Conference, May 20-25 2012, New Orleans LA, USA, THPPP057

[14] Y. Eidelman, Sputtering of Materials for the MEBT Absorber and PXIE Beam Dump, 2012, Project X Document 1091

[15] V.T. Astrelin et al, Blistering of selected materials irradiated by intense 200keV proton beam, Journal of Nuclear Materials 396 (2010) pp. 43-48

[16] L. Weissman et al., The use of a commercial copper beam dump for intense MeV proton beams Journal of Instrumentation 6 (2011) T03001

[17] S. Heifets, Q. Qin, and M. Zolotarev, Life of dust macroparticles in storage rings, PRST-AB, 8, 061002 (2005)

[18] S.K. Das, M. Kaminsky and P. Dusza, Surface damage of molybdenum and TZM alloy under D+ impact, Journal of Vacuum Science and Technology 15 (1978)

[19] Y. Nakamura, T. Shibata and M. Tanaka, Grain ejection from the surface of polycrystalline Molybdenum irradiated by intense H+ and H2+ ion beams, Journal of Nuclear Materials 68 (1977)

[20] F. Incropera and D. DeWitt, Fundamentals of heat and mass transfer, Fourth Edition, 1996

[21] M. Hassan et al., Analysis of the MEBT Beam dump, 2011, Project X Document 928

[22] ANSYS Mechanical Finite Element Analysis- http://www.ansys.com/

[23] P. Karditsas and M. Baptiste, Thermal and structural properties of fusion related materials, United Kingdom Atomic Energy Authority. Government Division, 1995, http://www-ferp.ucsd.edu/LIB/PROPS/PANOS/moa.html

[24] CASINO, a Monte Carlo code from Université de Sherbrooke, Québec, Canada, http://www.gel.usherbrooke.ca/casino/index.html

[25] ImageJ, available at http://rsbweb.nih.gov/ij/

[26] J. P. Waldront and D. W. Juenker, Optical Properties of Clean Molybdenum, Journal of the Optical Society of America, 54 (1964), pp. 204-207

[27] C. Baffes, A. Denisov, and A. Shemyakin, Feasibility of a radiation-cooled scraper for the PXIE $M E B T$, technical note, 2013, Project X Document 1221 